This is an electronic reprint of the original article. This reprint may differ from the original in pagination and typographic detail.

Author(s): Perez-Ramos, Redamy; Renk, Thorsten

Title: $\quad$ In-medium jet shape from energy collimation in parton showers: Comparison with CMS PbPb data at 2.76 TeV

Year: $\quad 2014$

Version:

Please cite the original version:

Perez-Ramos, R., \& Renk, T. (2014). In-medium jet shape from energy collimation in parton showers: Comparison with CMS PbPb data at 2.76 TeV. Physical Review D, 90(1), Article 014018. https://doi.org/10.1103/PhysRevD.90.014018

All material supplied via JYX is protected by copyright and other intellectual property rights, and duplication or sale of all or part of any of the repository collections is not permitted, except that material may be duplicated by you for your research use or educational purposes in electronic or print form. You must obtain permission for any other use. Electronic or print copies may not be offered, whether for sale or otherwise to anyone who is not an authorised user. 
PHYSICAL REVIEW D 90, 014018 (2014)

\title{
In-medium jet shape from energy collimation in parton showers: Comparison with CMS PbPb data at 2.76 TeV
}

\author{
Redamy Pérez-Ramos* \\ Department of Physics, University of Jyväskylä, P.O. Box 35, Jyväskylä FI-40014, Finland \\ Thorsten Renk ${ }^{\dagger}$ \\ Department of Physics, University of Jyväskylä, P.O. Box 35, Jyväskylä FI-40014, Finland \\ and Helsinki Institute of Physics, University of Helsinki, P.O. Box 64, Helsinki FI-00014, Finland
}

(Received 17 February 2014; published 10 July 2014)

\begin{abstract}
We present the medium-modified energy collimation in the leading-logarithmic approximation (LLA) and next-to-leading-logarithmic approximation (NLLA) of QCD. As a consequence of more accurate kinematic considerations in the argument of the Dokshitzer-Gribov-Lipatov-Altarelli-Parisi (DGLAP) fragmentation functions (FFs) we find a new NLLA correction $\mathcal{O}\left(\alpha_{s}\right)$ which accounts for the scaling violation of DGLAP FFs at small $x$. The jet shape is derived from the energy collimation within the same approximations and we also compare our calculations for the energy collimation with the event generators PYTHIA6 and YAJEM for the first time in this paper. The modification of jets by the medium in both cases is implemented by altering the infrared sector using the Borghini-Wiedemann model. The energy collimation and jet shapes qualitatively describe a clear broadening of showers in the medium, which is further supported by YAJEM in the final comparison of the jet shape with CMS PbPb data at center-of-mass energy 2.76 TeV. The comparison of the biased versus unbiased YAJEM jet shape with the CMS data shows a more accurate agreement for biased showers and illustrates the importance of an accurate simulation of the experimental jet-finding strategy.
\end{abstract}

DOI: 10.1103/PhysRevD.90.014018

PACS numbers: 12.38.-t, 12.38.Mh

\section{INTRODUCTION}

The phenomenon of jet quenching was first established experimentally through the observed suppression of high$p_{T}$ hadrons in nucleus-nucleus (A-A) collisions at the Relativistic Heavy Ion Collider (RHIC) and the Large Hadron Collider (LHC) [1-4]. It was then confirmed by various other measurements where highly virtual partons produced in hard processes in a medium showed modifications to the subsequent evolution of a QCD shower, in particular a softening and broadening of the resulting hadron distribution which leads to a reduction in the yield of leading hadrons and jets [5-9].

In the vacuum, the production of highly virtual partons following the hard inelastic scattering of two partons from the incoming protons $(2 \rightarrow 2+X)$ is followed by the fragmentation into a spray of hadrons which are observed in high-energy collider experiments. The evolution of successive splittings $q(\bar{q}) \rightarrow q(\bar{q}) g, g \rightarrow g g$ and $g \rightarrow q \bar{q}$ $(q, \bar{q}$ and $g$ label quark, antiquark and gluon, respectively) inside the parton shower prior to hadronization is well established and can be described by the Dokshitzer-GribovLipatov-Altarelli-Parisi (DGLAP) evolution equations for fragmentation functions in the leading-logarithmic approximation (LLA) of QCD [10-12] or alternatively in terms of

\footnotetext{
redamy.r.perez-ramos@jyu.fi

†trenk@phy.duke.edu
}

Monte Carlo (MC) formulations such as the PYSHOW algorithm [13,14]. In A-A collisions, partons produced in the hard inelastic scattering of two partons from nuclei $(2 \rightarrow 2+X)$ propagate through the hot/dense QCD media also produced in such collisions and their branching pattern is changed by interacting with the color charges of the deconfined quark-gluon plasma (QGP) [15]. As a consequence, additional medium-induced soft gluon radiation is produced in A-A collisions, which leads for instance to the modification of high- $p_{T}$ hadroproduction [16-18]. At RHIC, the main observables considered to probe this physics were the nuclear suppression factor of single inclusive hadrons $R_{A A}[19,20]$ and the suppression factor of hard back-to-back dihadron correlations $I_{A A}$ [21,22]. More recently, through the analytical developments of Refs. [23-25], it was demonstrated that medium-induced soft gluon radiation is ruled by a condition of antiangular ordering over successive emissions of such gluons, which oppositely to the condition of angular ordering in the vacuum (for a review see Ref. [26]), leads to the jet broadening. However, further efforts are required in order to implement the full description of color decoherence effects in Monte Carlo event generators.

In this paper, we aim at discussing two different observables currently relevant mainly for LHC physics: the energy collimation and the jet shape of mediummodified showers. We use a QCD-inspired model introduced by Borghini and Wiedemann (BW) [27] for the 
modification of the fragmentation functions (FFs) by the medium where the medium evolution itself is described by a hydrodynamical evolution. In the BW model, the DGLAP splitting functions are enhanced in the infrared sector in order to mimic the medium-induced soft gluon radiation. In practice, the $1 / z$ dependence of the $Q C D$ vacuum splitting functions corresponding to the parton branchings $q(\bar{q}) \rightarrow q(\bar{q}) g$ and $g \rightarrow g g$ are altered by introducing a parameter $N_{\mathrm{s}}=1+f_{\text {med }}\left(f_{\text {med }} \geq 0\right)$ in the form $P_{a \rightarrow b c}(z)=N_{s} / z+\mathcal{O}(1)$, which is simple and mostly leads to analytical results. In Ref. [28], which appeared long after Ref. [27], Nayak derived in-medium expressions for the quark and gluon DGLAP splitting functions in nonequilibrium (nonisotropic) QCD at leading order in $\alpha_{s}$ as a function of arbitrary nonequilibrium distribution functions $f_{q}(\vec{p})$ and $f_{g}(\vec{p})$, where $\vec{p}$ is the momentum of the hard parton. The modification to the splitting functions turns out to be quite similar to the one introduced in the BW model [27] by some prefactor depending on $f_{q}(\vec{p})$ and $f_{g}(\vec{p})$, which affects both the infrared and regular terms of the evolution kernels. Later on, in Refs. [25,29], the splitting functions were modified by an overall factor which depends on the pertinent medium parameters, such as the medium transport coefficient $\hat{q}$. Whether these modification prefactors are related to those found in Ref. [28] may be an interesting issue of further investigation, but this is out of the scope of this paper. Moreover, the BW model in Ref. [27] and the calculations in Ref. [29] show that the production of soft hadrons as described by the FFs is enhanced at small energy fraction $x$ of the outgoing hadrons. The same result was also found in Ref. [30], where a full resummation from large to small $x$ was performed in the same frame of the vacuum AlbinoKniehl-Kramer parametrization of FFs (for a detailed review see Ref. [31]), which further motivates the use of the simple BW prescription in the following.

We start with the quantification of the jet energy collimation and a study of the jet broadening in gluon and quark jets in LLA. The computation of the energy collimation was first performed analytically in the vacuum [32] and subsequently modeled in the medium [33] by means of the inclusive spectrum of partons provided by the medium-modified solution of DGLAP FFs at large $x \sim 1$. For this purpose, a high-energy jet of half opening angle $\Theta_{0}$, energy $E$ and virtuality $Q=E \Theta_{0}$ produced in a nucleus-nucleus collision was considered, followed by the production of one concentric sub-jet of opening angle $\Theta$ and transverse momentum $k_{\perp}=x E \Theta$ where the bulk $x E \sim E$ of the jet energy is contained [32,33]. By definition, the smaller the angle $\Theta$ where the jet energy is concentrated, the higher the jet energy collimation [32]. The half opening angle of the jet $\Theta_{0}$ should be fixed according to the jet definition used by the experiment. The energy collimation can be then determined by maximizing the distribution of partons $D\left(x, E \Theta_{0}, E \Theta\right)$, which dominates the hard fragmentation $(x \sim 1)$ of the jet into a sub-jet, as discussed in Ref. [33]. In this paper, we will provide a more accurate description of the energy collimation, which accounts for the $x$ dependence in the third argument of the FFs $D\left(x, \ln E \Theta_{0}, \ln x E \Theta\right)$. The account of the shift in $\ln x$ leads to a small next-to-leading-logarithmic approximation (NLLA) correction of order $\mathcal{O}\left(\alpha_{s}\right)$ which decreases the energy collimation at intermediate values of $x$ as a consequence of the scaling violation of DGLAP FFs [34]. Our first aim is indeed to make a comparison for the jet energy collimation between the LLA and NLLA with PYTHIA6 [13,14] and YAJEM [35] in the medium. Such studies have been done for the vacuum case [36], but it is far from being evident that there are no additional differences in the medium and this issue should be further studied.

The integrated jet shape $\Psi\left(\Theta ; \Theta_{0}\right)$ provides indeed an analogous measurement of how widely the transverse energy of the jet is spread. This observable was first studied in the vacuum in Ref. [36] and generalized to the medium throughout the calculations presented in Refs. [37,38] in the framework of the cone and $k_{t}$ jet reconstruction algorithms. By definition, $\Psi\left(\Theta ; \Theta_{0}\right)$ determines the energy fraction $\left[x \equiv \Psi\left(\Theta ; \Theta_{0}\right)\right]$ of a jet of half opening angle $\Theta_{0}$ that falls into a sub-jet of half opening angle $\Theta$ for a fixed jet energy $E$. In our framework, we extract for the first time the angular dependence of the subjet energy fraction from the simple prescription provided by the LLA DGLAP energy collimation $x=f\left(E \Theta_{0}, E \Theta\right)$ at fixed energy $E$; no other computation for this observable is known in the context of LLA DGLAP evolution equations. Thus, the jet energy collimation is a LLA DGLAP observable; however, it is not measured in a heavy-ion collisions context, but jet shapes are. That is why, in the comparison with $\mathrm{MC}$, we do the jet shape analysis with PYTHIA6 and YAJEM using the FASTJET package $[39,40]$ on the events and compare our results with CMS $p p$ and $\mathrm{PbPb}$ data for central collisions $(0-10 \%)$ at $2.76 \mathrm{TeV}[8]$. The BW prescription provides a simple test case for this; as it is analytically solvable and easily implemented in the YAJEM code, it will be shown to capture the main physics of additional soft gluon production and jet broadening through this observable.

Finally, for the purpose of a detailed comparison with data from the CMS experiment, the jet-shape analysis with YAJEM is performed for both $\mathrm{PbPb}$ and $p p$ collisions following the CMS analysis procedure closely. Jets are reconstructed with the anti- $k_{T}$ algorithm $[39,40]$ with a resolution parameter $R\left(\equiv \Theta_{0}\right)=0.3$. The clustering analysis is limited to charged particles with $e_{i}>1 \mathrm{GeV}$ inside the jet cone where $E_{\mathrm{rec}} \geq 100 \mathrm{GeV}$ is required for a jet (i.e. $E_{\text {rec }}$ stands for the recovered jet energy inside the cone) [8]. The condition $e_{i}>1 \mathrm{GeV}$ removes the soft QCD medium background which may blur the jet fragmentation and jetshape analysis. In order to illustrate the role of the bias 
caused by the jet-finding procedure outlined above, we compare the biased jet shape (i.e. provided the CMS jetfinding conditions are fulfilled) with an unbiased jet shape (which is a purely theoretical quantity) for both $\mathrm{PbPb}$ and $p p$ CMS data.

\section{THEORETICAL FRAMEWORK}

\section{A. Description of the process and kinematics}

In Fig. 1, we consider the production of one gluon or quark $(A=g, q, \bar{q})$ jet of total energy $E$ and opening angle $\Theta_{0}$ which fragments into a sub-jet $B$ of energy $x E$ and opening angle $\Theta\left(\Theta \leq \Theta_{0}\right)$, where $x$ is the energy fraction of $A$ carried by $B$.

By definition, the virtualities of the jet $A$ and the sub-jet $B$ are $Q=E \Theta_{0}$ and $k_{\perp}=E_{p} \Theta\left(E_{p}=x E\right)$, respectively. The virtuality, also known as the hardness of the jet, determines the phase space for radiation and hence sets the maximal transverse momentum of a parton inside the jet: $k_{\perp} \leq Q$. A minimal cutoff parameter $Q_{0}$ can be introduced $k_{\perp} \geq Q_{0}$, such that the minimal angle reached by a parton inside the cascade equals $\Theta_{\min } \geq Q_{0} / x E \Theta_{0}$. Experimentally, this physical picture corresponds to the calorimetric measurement of the energy flux deposited within a given solid angle. From the partonic point of view, the successive decays of partons in the cascade are ordered in $k_{\perp, i}$, or angles $\Theta_{i}$ due to the LLA kinematics for hard parton decays $(x \sim 1)$ or due to the QCD coherence for soft parton decays $(x \ll 1)$ [32]. Hard parton decays determine the bulk of the jet energy and are ruled by the LLA kinematics, which leads to DGLAP evolution equations [41], while soft parton decays determine the bulk of the jet multiplicity and are ruled by QCD coherence, which leads instead to the modified-LLA (MLLA) evolution equations [32].

The jet energy collimation is characterized by the large energy fraction $x$ of the sub-jet where the bulk of the jet energy inside the given cone $\Theta<\Theta_{0} \ll 1$ is deposited. Hence, the probability for the energy fraction $x$ to be deposited in a cone of aperture $\Theta$ is related to the DGLAP inclusive spectrum of partons through the formula [42],

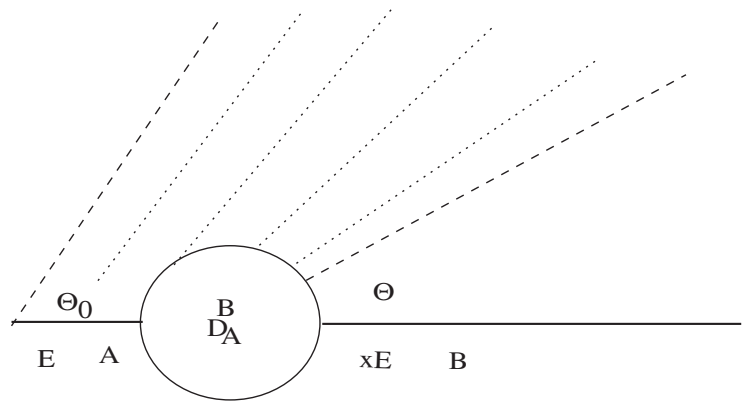

FIG. 1. Fragmentation of a jet $A$ of half opening angle $\Theta_{0}$ into a sub-jet $B$ of half opening angle $\Theta<\Theta_{0}$.

$$
D_{A}\left(x, E \Theta_{0}, x E \Theta\right)=\sum_{B=g, q} D_{A}^{B}\left(x, E \Theta_{0}, x E \Theta\right),
$$

where the nature of partons $B$ is not identified. In Eq. (1), the FFs $D_{A}^{B}\left(x, E \Theta_{0}, x E \Theta\right)$ determine the probability that a parton $A$ produced at large $p_{T} \sim E$ in a high-energy collision fragments into a hard sub-jet $B$ of transverse momentum $x E \Theta$, which we write in the third argument of the FF. Qualitatively, Eq. (1) describes the evolution of the jet $A$ in the $k_{\perp}$ range $x E \Theta \leq k_{\perp} \leq E \Theta_{0}$ according to the LLA $k_{\perp}$ ordering and hence, it determines the partonic skeleton of the sub-jet $B$ before the hadronization takes place.

As compared to the FF for the inclusive spectrum of partons where the third argument is set to $E \Theta$ for hard partons $x \sim 1: D_{A}^{B}\left(x, E \Theta_{0}, E \Theta\right)$ [33], the formula (1) accounts for the energy fraction $x$ of the sub-jet $B$ in the FFs $D_{A}^{B}\left(x, E \Theta_{0}, x E \Theta\right)$. We cannot compute $D_{A}^{B}\left(x, E \Theta_{0}, x E \Theta\right)$ by using DGLAP evolution equations because of the $x$ dependence included in the third argument, but we can instead expand it in powers of "ln $x$ " through the exponential operator,

$$
D_{A}^{B}\left(x, E \Theta_{0}, x E \Theta\right)=e^{\ln x(\partial / \partial \ln (E \Theta))} D_{A}^{B}\left(x, E \Theta_{0}, E \Theta\right)
$$

such that,

$$
\begin{aligned}
& D_{A}^{B}\left(x, E \Theta_{0}, x E \Theta\right) \\
& =D_{A}^{B}(x, \Delta \xi)-\ln x \frac{\alpha_{s}\left(E \Theta_{0}\right)}{2 \pi} e^{4 N_{c} \beta_{0} \Delta \xi} \frac{\partial D_{A}^{B}}{\partial \Delta \xi}(x, \Delta \xi) \\
& \quad+\mathcal{O}\left(\alpha_{s}^{2}\right),
\end{aligned}
$$

where,

$$
\begin{aligned}
\xi(E \Theta) & =\frac{1}{4 N_{c} \beta_{0}} \ln \left[\ln \left(\frac{E \Theta}{\Lambda_{\mathrm{QCD}}}\right)^{2}\right], \\
\Delta \xi & =\xi\left(E \Theta_{0}\right)-\xi(E \Theta), \quad \alpha_{s}\left(E \Theta_{0}\right)=\frac{2 \pi}{4 N_{c} \beta_{0} \ln \left(\frac{E \Theta_{0}}{\Lambda_{\mathrm{QCD}}}\right)} .
\end{aligned}
$$

Hence, Eq. (1) can be rewritten in the form,

$$
\begin{aligned}
& D_{A}\left(x, E \Theta_{0}, x E \Theta\right) \\
& =\sum_{B=g, q}\left[D_{A}^{B}(x, \Delta \xi)-\ln x \frac{\alpha_{s}\left(E \Theta_{0}\right)}{2 \pi} e^{4 N_{c} \beta_{0} \Delta \xi} \frac{\partial D_{A}^{B}}{\partial \Delta \xi}(x, \Delta \xi)\right] \\
& \quad+\mathcal{O}\left(\alpha_{s}^{2}\right),
\end{aligned}
$$

where $\alpha_{s}$ is the QCD coupling constant, $\beta_{0}$ is the first coefficient of the QCD $\beta$ function given by $\beta_{0}=$ $\frac{1}{4 N_{c}}\left(\frac{11}{3} N_{c}-\frac{4}{3} T_{R} n_{f}\right)$, with $N_{c}=3, T_{R}=\frac{1}{2}, n_{f}=3$ and $\Lambda_{\mathrm{QCD}}(=300 \mathrm{MeV})$ is the mass scale of QCD. The new correction $\mathcal{O}\left(\alpha_{s}\right)$ is very small as $x \rightarrow 1$ and can be much 
larger for $x \approx 0.5$. As displayed in Fig. 1, the ladder Feynman diagrams leading to DGLAP evolution equations for $D_{A}^{B}(x, \Delta \xi)$ should be iterated from the hardest virtuality $Q=E \Theta_{0}$ of the process to the lower sub-jet virtuality $k_{\perp}=E \Theta$ through the variable $\Delta \xi$ in Eq. (4). Thus, $D_{A}^{B}(x, \Delta \xi)$ describes the distribution of partons $B$ with transverse momentum $k_{\perp}=E \Theta$ contained inside the parton $A$, which fixes the initial scale of the hard process: $Q=E \Theta_{0}$; i.e. the virtuality. Therefore, we can estimate $D_{A}\left(x, E \Theta_{0}, x E \Theta\right)$ with the solution of DGLAP evolution equations for the FFs $D_{A}^{B}(x, \Delta \xi)$, which appear on the rhs of Eq. (5).

\section{B. Medium-modified DGLAP evolution equations with the BW model}

The DGLAP evolution equations for the splitting $A[1] \rightarrow$ $B[z] C[1-z]$ (where $z$ is the energy fraction of one parton in the splitting) in the $k_{\perp}$ range $E \Theta \leq k_{\perp} \leq E \Theta_{0}$ takes the simple form [41]

$$
\begin{aligned}
& \frac{d}{d \ln E \Theta} D\left(x, E \Theta_{0}, E \Theta\right) \\
& \quad=\frac{\alpha_{s}(E \Theta)}{4 \pi} \int_{x}^{1} \frac{d z}{z} P(z) D\left(\frac{x}{z}, E \Theta_{0}, E \Theta\right),
\end{aligned}
$$

where $P(z)$ is the evolution "Hamiltonian" given by the regularized splitting functions [10-12]. In order to account for the medium-induced soft gluon radiation in heavy-ion collisions, we make use of the QCD-inspired model proposed in Ref. [27] which leads to a simple solution of the evolution equations at $x \sim 1$. In this model, the infrared parts of the splitting functions are arbitrarily enhanced by the factor $N_{s}=1+f_{\text {med }}$, where $f_{\text {med }} \geq 0$ accounts for medium-induced soft gluon radiation.
The medium-modified splitting functions in $z$ space are written in the form [27],

$$
\begin{aligned}
P_{g g}(z)= & 4 N_{c}\left[\frac{N_{s}}{z}+\left[\frac{N_{s}}{1-z}\right]_{+}+z(1-z)-2\right], \\
P_{g q}(z)= & 2 T_{R}\left[z^{2}+(1-z)^{2}\right], \\
& P_{q g}(z)=2 C_{F}\left(\frac{2 N_{s}}{z}+z-2\right), \\
& P_{q q}(z)=2 C_{F}\left(\left[\frac{2 N_{s}}{1-z}\right]_{+}-1-z\right),
\end{aligned}
$$

where the $[\ldots]_{+}$prescription is defined as $\int_{0}^{1} d x[F(x)]_{+} g(x) \equiv \int_{0}^{1} d x F(x)[g(x)-g(1)]$. The solutions of Eq. (6) can most conveniently be obtained in Mellin space $\mathcal{D}\left(j, E \Theta_{0}, E \Theta\right)$ through the transformation

$$
\mathcal{D}\left(j, E \Theta_{0}, E \Theta\right)=\int_{0}^{1} d x x^{j-1} D\left(x, E \Theta_{0}, E \Theta\right),
$$

such that the convolution (6) yields,

$$
\begin{aligned}
\frac{d}{d \ln E \Theta} \mathcal{D}\left(j, E \Theta_{0}, E \Theta\right) & =\mathcal{P}(j) \mathcal{D}\left(j, E \Theta_{0}, E \Theta\right), \\
\mathcal{P}(j) & =\int_{0}^{1} d z z^{j-1} P(z) .
\end{aligned}
$$

The advantage of the Mellin transform can be clearly seen in Eq. (8). The convolution over $z$ in Eq. (6) reduces to the product of the Mellin-transformed splitting functions $\mathcal{P}(j)$ and the FFs $\mathcal{D}\left(j, E \Theta_{0}, E \Theta\right)$. Making use of the variables introduced in Eq. (4), Eq. (8) can be more explicitly rewritten in the matrix form at leading order (LO),

$$
\frac{d}{d \xi}\left(\begin{array}{c}
\mathcal{D}_{q_{\mathrm{NS}}}(j, \xi) \\
\mathcal{D}_{q_{\mathrm{s}}}(j, \xi) \\
\mathcal{D}_{g}(j, \xi)
\end{array}\right)=\left(\begin{array}{ccc}
\mathcal{P}_{q q}(j) & 0 & 0 \\
0 & \mathcal{P}_{q q}(j) & \mathcal{P}_{q g}(j) \\
0 & \mathcal{P}_{g q}(j) & \mathcal{P}_{g g}(j)
\end{array}\right)\left(\begin{array}{c}
\mathcal{D}_{q_{\mathrm{NS}}}(j, \xi) \\
\mathcal{D}_{q_{\mathrm{s}}}(j, \xi) \\
\mathcal{D}_{g}(j, \xi)
\end{array}\right)
$$

where $\mathcal{D}_{q_{\mathrm{NS}}}$ and $\mathcal{D}_{q_{\mathrm{s}}}$ stand, respectively, for the flavornonsinglet and flavor-singlet quark distributions, and $\mathcal{P}_{i k}(j)$ are the Mellin transforms of the LO splitting functions:

$$
\begin{aligned}
& \mathcal{P}_{g g}(j)=-4 N_{c}\left[N_{s} \psi(j+1)+N_{s} \gamma_{E}-\frac{N_{s}-1}{j}-\frac{N_{s}-1}{j-1}\right] \\
&+ \frac{11 N_{c}}{3}-\frac{2 n_{f}}{3}+\frac{8 N_{c}\left(j^{2}+j+1\right)}{j\left(j^{2}-1\right)(j+2)}, \\
& \mathcal{P}_{g q}(j)=T_{R} \frac{j^{2}+j+2}{j(j+1)(j+2)},
\end{aligned}
$$

$$
\begin{aligned}
& \mathcal{P}_{q g}(j)=2 C_{F} \frac{\left(2 N_{s}-1\right)\left(j^{2}+j\right)+2}{j\left(j^{2}-1\right)}, \\
\mathcal{P}_{q q}(j)= & -C_{F}\left[4 N_{s} \psi(j+1)+4 N_{s} \gamma_{E}-4 \frac{N_{s}-1}{j}\right. \\
& \left.-3-\frac{2}{j(j+1)}\right] .
\end{aligned}
$$

This method allows for the diagonalization of the "Hamiltonian" given by the set $\mathcal{P}(j)$ with respect to the "evolution-time" variable $\xi \sim t=\ln (E \Theta)$. In some limits at large and small $x$, analytical solutions of the equations 
can be found through this method [41] but for numerical computation, solving the equations directly in $x$ space turns out to be more efficient than inverting the Mellin transform numerically. Thus, at large energy fraction $x \sim 1$, or equivalently large $j \gg 1$ (which we are interested in), the expressions for the Mellin representation of the splitting functions (10a)-(10d) can be reduced to

$$
\begin{aligned}
& \mathcal{P}_{q q}(j) \approx 4 C_{F} N_{s}\left(-\ln j+\frac{3}{4 N_{s}}-\gamma_{E}\right), \\
& \mathcal{P}_{g g}(j) \approx 4 N_{c} N_{s}\left(-\ln j+\frac{\beta_{0}}{N_{s}}-\gamma_{E}\right),
\end{aligned}
$$

where the asymptotic behavior of the digamma function $\psi(j+1) \approx \ln j$ is replaced at $j \gg 1$ [41]. The off-diagonal matrix elements vanish in this approximation: $\mathcal{P}_{g q}(j)=\mathcal{P}_{q g}(j)=0$.

Note that the $N_{s} \ln j$ dependence in Eq. (11) arises from the $\left[N_{s} /(1-z)\right]_{+}$terms of the DGLAP splitting functions, such that for hard partons $z \sim 1$, the enhanced contribution of the soft $1-z \sim 0$ component $[1 /(1-z)]_{+}$produces the sub-jet broadening within this approximation. Qualitatively, as a consequence of energy conservation, if soft gluon radiation is enhanced in the region $\Theta \leq \Theta^{\prime} \leq \Theta_{0}$ for a fixed jet energy $E$, the sub-jet energy $(B, x E)$ should be smaller compared to its value in the vacuum and the energy collimation should then decrease, i.e. $\Theta$ should increase.

Going back to $x$ space requires taking the inverse Mellin transform given by

$$
D(x, \Delta \xi)=\frac{1}{2 \pi i} \int_{C} d j x^{-j} \mathcal{D}(j, \Delta \xi),
$$

where the contour $C$ in the complex plane is parallel to the imaginary axis and lies to the right of all singularities. Since we are interested in the large $j \gg 1(x \sim 1)$ approximation, we insert Eq. (11) into Eq. (9). After integrating the result, we get the medium-modified distribution at large $x \sim 1$,

$$
D_{A}^{A}(x, \Delta \xi) \simeq(1-x)^{-1+4 C_{A} N_{s} \Delta \xi} \frac{\exp \left[4 C_{A} N_{s}\left(\frac{3}{4 N_{s}}-\gamma_{E}\right) \Delta \xi\right]}{\Gamma\left(4 C_{A} N_{s} \Delta \xi\right)},
$$

where $\beta_{0}$ is replaced by $3 / 4\left(n_{f}=3\right)$ in Eq. (11). The corresponding result in the vacuum for $N_{s}=1$ is given in Ref. [41].

Within this approximation, the parton initiating the jet $A$ is identical to that initiating the sub-jet $B=A, C_{A}=N_{c}$ if $A$ is a gluon and $C_{A}=C_{F}=\frac{4}{3}$ if $A$ is a quark. Indeed, the FF $D_{A}^{A}(x, \Delta \xi)$ in Eq. (13) describes the splittings $g \rightarrow g g$ and $q \rightarrow q g$ and as constructed, it neglects the others: $g \rightarrow q \bar{q}$ and $q \rightarrow g q$. Therefore, the sum over $B$ in Eq. (5) disappears such that,

$$
\begin{aligned}
& D_{A}^{A}\left(x, E \Theta_{0}, x E \Theta\right) \\
& =D_{A}^{A}(x, \Delta \xi)-\ln x \frac{\alpha_{s}\left(E \Theta_{0}\right)}{2 \pi} e^{4 N_{c} \beta_{0} \Delta \xi} \frac{\partial D_{A}^{A}}{\partial \Delta \xi}(x, \Delta \xi) \\
& \quad+\mathcal{O}\left(\alpha_{s}^{2}\right) .
\end{aligned}
$$

In a more accurate solution of this problem which could only be achieved numerically, the whole sum of the parton branchings is given by $D_{q}\left(x, E \Theta_{0}, x E \Theta\right)=$ $D_{q}^{q}\left(x, E \Theta_{0}, x E \Theta\right)+D_{q}^{g}\left(x, E \Theta_{0}, x E \Theta\right)$ for a quark jet and $D_{g}\left(x, E \Theta_{0}, x E \Theta\right)=D_{g}^{g}\left(x, E \Theta_{0}, x E \Theta\right)+D_{g}^{q}\left(x, E \Theta_{0}, x E \Theta\right)$ for a gluon jet, with the full resummed contribution of the soft gluon/collinear logarithms arising from the $N_{s} / z$ dependence of the splitting functions in the FO approach of DGLAP FFs $[30,43]$.

\section{Jet energy collimation}

As discussed in Ref. [41], the distribution (13) presents a certain maximum at some angle $\Theta$ where the bulk of the jet energy is concentrated. The reason for this can be understood as follows: for $\Delta \xi \rightarrow 0$, or $\Theta \rightarrow \Theta_{0}$, almost all of the energy is contained inside the cone $\Theta_{0}$ [i.e. $D \rightarrow \delta(1-x)$ ] and the probability distribution $D_{A}^{A}$ for $x \neq 1$ should decrease. For $\Theta$ decreasing $\Theta \gg \Lambda_{\mathrm{QCD}} / E$ [notice that the $x$ dependence was reabsorbed on the pre-exponential term in Eq. (2)], the emission outside the cone $\Theta$ grows and the fragmentation probability decreases. Then, taking the first derivative over $\ln \Theta$ in Eq. (14) leads to the NLLA (not to be confused with the MLLA) equation for $\Theta$ :

$$
\begin{aligned}
& {\left[\ln (1-x)+\frac{3}{4 N_{s}}-\gamma_{E}-\psi\left(4 C_{A} N_{s} \Delta \xi\right)\right]\left(1-4 N_{c} \beta_{0} e^{b \Delta \xi} \ln x \frac{\alpha_{s}\left(E \Theta_{0}\right)}{2 \pi}\right)} \\
& =4 C_{A} N_{s} e^{4 N_{c} \beta_{0} \Delta \xi} \ln x \frac{\alpha_{s}\left(E \Theta_{0}\right)}{2 \pi}\left[\ln (1-x)+\frac{3}{4 N_{s}}-\gamma_{E}-\psi\left(4 C_{A} N_{s} \Delta \xi\right)\right]^{2} \\
& \quad-4 C_{A} N_{s} e^{4 N_{c} \beta_{0} \Delta \xi} \ln x \frac{\alpha_{s}\left(E \Theta_{0}\right)}{2 \pi} \psi^{(1)}\left(4 C_{A} N_{s} \Delta \xi\right),
\end{aligned}
$$


which is the main theoretical result of this section for medium $\left(N_{s}>1\right)$ and also vacuum $\left(N_{s}=1\right)$. We invert Eq. (15) numerically in order to get the NLLA jet energy collimation $\Theta(x, E)$. In Eq. (15), $\psi(x)$ is the digamma function and $\psi^{(1)}(x)=\frac{d \psi(x)}{d x}$ is the polygamma function of the first order, which is new in this context. Note that this is one correction; a more complete set of corrections of the same order can be also added if, for instance, one considers the next-to-leading-order corrections [44] to the approached splitting functions (11) in a more cumbersome approach of this problem. However, this term goes beyond DGLAP and corresponds to the so-called scaling violation in DGLAP fragmentation functions [34]. In our framework, this correction slightly increases the available phase space from the hardest $(B, x \sim 1)$ to slightly softer partons $(B, x \sim 0.5)$ and is therefore expected to decrease the energy collimation or increase $\Theta$ at intermediate $x$. As expected for harder partons $\ln x \sim 0$, the above equation (15) reduces to the simpler one [33],

$$
\psi\left(4 C_{A} N_{s} \Delta \xi\right)=\ln (1-x)+\frac{3}{4 N_{s}}-\gamma_{E} .
$$

Symbolically, the inversion of the NLLA (15) and LLA (16) can be written for quark $(A=q, \bar{q})$ and gluon $(A=g)$ jets in the simple form,

$$
\frac{\Theta_{A}}{\Theta_{0}}=\left(\frac{E \Theta_{0}}{\Lambda_{\mathrm{QCD}}}\right)^{-\gamma_{A}\left(x, N_{s}\right)} .
$$

Setting $\ln x \rightarrow 0$ in Eq. (15), the LLA expression for $\gamma_{A}\left(x, N_{s}\right)$ is simply written in the form [33]

$\gamma_{A}\left(x, N_{s}\right)=1-\exp \left[-\frac{N_{c} \beta_{0}}{C_{A} N_{s}} \psi^{-1}\left(\ln (1-x)+\frac{3}{4 N_{s}}-\gamma_{E}\right)\right]$,

where $\psi^{-1}$ is the inverse of the digamma function. The exponent $\gamma_{A}\left(x, N_{S}\right)$ provides indeed the medium-modified slope of the energy collimation as a function of $N_{s}$ for a fixed value of the sub-jet energy fraction $x$ and can be obtained numerically from the NLLA equation (15). In Table I, we display the values of the NLLA and LLA slopes for $x=0.5$ and $x=0.8$, which are in agreement with the LLA (NLLA) DGLAP large sub-jet energy fraction $x$ approximation where these predictions should be tested.

TABLE I. NLLA and LLA values of the slope $\gamma_{A}\left(x, N_{s}\right)$ of the energy collimation for $N_{s}=1.4$ (medium) and $N_{s}=1$ (vacuum).

\begin{tabular}{lccccc}
\hline NLLA, LLA & $x=0.5$ & $x=0.8$ & NLLA, LLA & $x=0.5$ & $x=0.8$ \\
\hline$\gamma_{g}(x, 1.4)$ & 0.37 & 0.26 & $\gamma_{g}(x, 1)$ & 0.54 & 0.38 \\
$\gamma_{q}(x, 1.4)$ & 0.67 & 0.50 & $\gamma_{q}(x, 1)$ & 0.83 & 0.65 \\
\hline \hline
\end{tabular}

As $x \rightarrow 0$, the fixed-order (FO) approach of the LLA fails to provide any reliable result.

The new equation (15) cannot be rewritten like Eq. (17) but it can be solved numerically. From Table I, one may wonder why the NLLA (15) and LLA (16) slopes of the energy collimation are the same. ${ }^{1}$ Indeed, the coupling constant does not depend on the jet energy only, but rather on the product $E \Theta \gg \Lambda_{\mathrm{QCD}}$ through the term $\ln x e^{4 N_{c} \beta_{0} \Delta \xi} \alpha_{s}\left(E \Theta_{0}\right) \sim \ln x \alpha_{s}(E \Theta)$ in Eq. (15). As the jet energy $E$ increases, the sub-jet cone $\Theta$ decreases and $\alpha_{s}(E \Theta)$ should remain roughly constant. Therefore, the NLLA and LLA curves of the jet energy collimation should stay approximately parallel to each other asymptotically.

We can see in both cases that the nuclear suppression parameter $N_{s}$ decreases the slope of the energy collimation, which translates into increasing the rate of the jet broadening asymptotically. In both medium and vacuum $\gamma_{q}>\gamma_{g}$, which physically means that quark jets are more collimated than gluon jets. The same trends should be confirmed in the forthcoming analysis of the jet energy collimation with the event generator YAJEM.

\section{COMPARISON WITH YAJEM AND QGP HYDRODYNAMICS}

In order to gauge the impact of the approximations made in deriving the results of the preceding section, we compare them with results for jet energy collimation obtained in a $\mathrm{MC}$ formulation of the in-medium jet evolution. Within such a model, the parton initiating a jet $A$ does not have to be identical to that initiating the sub-jet $B$ and hence the full set of splittings $g \rightarrow g g$ and $g \rightarrow q \bar{q}$ is available for a gluon jet. In addition, exact energy-momentum conservation at every splitting vertex is enforced.

In vacuum, the PYSHOW algorithm $[13,14]$ is a welltested numerical implementation of QCD shower simulations. For comparison with our analytic results, we use the Borghini-Wiedemann prescription implemented within the in-medium shower code [45].

\section{A. The in-medium shower generator YAJEM}

YAJEM is based on the PYSHOW algorithm, to which it reduces in the limit of no medium effects. It simulates the evolution of a QCD shower as an iterated series of splittings of a parent into two daughter partons $a \rightarrow b c$ where the energy of the daughters are obtained as $E_{b}=z E_{a}$ and $E_{c}=$ $(1-z) E_{a}$ and the virtuality of the parent and daughters is ordered as $Q_{a} \gg Q_{b}, Q_{c}$. The decreasing hard virtuality scale of partons provides (splitting by splitting) the transverse phase space for radiation, and the perturbative QCD evolution terminates once the parton virtuality

\footnotetext{
${ }^{1}$ Notice that Table I displays indeed 16 values for the slopes, but such numbers are identical for the NLLA and LLA energy collimation.
} 
reaches a lower value $Q_{0}=1 \mathrm{GeV}$, at which point the subsequent evolution is considered to be nonperturbative hadronization.

The probability distribution to split at given $z$ is given by the same QCD splitting kernels and their medium modification in the BW prescription, which we have used above, i.e. Eqs. (7a) and (7b); however, in the explicit kinematics of the MC shower the singularities for $z \rightarrow 0$ or $z \rightarrow 1$ are outside of accessible phase space and no $[\ldots]_{+}$regularization procedure is needed.

We will refer to the implementation of the BW prescription for in-medium showers in the following as YAJEM+BW (note that this corresponds to the FMED scenario described in Ref. [45]). This is distinct from the default version of the code YAJEM, YAJEM-DE, which is tested against multiple observables at both RHIC and LHC (see e.g. Refs. [46-48]) and is based on an explicit exchange of energy and momentum between jet and medium rather than a modification of splitting probabilities.

For a straightforward benchmark comparison with analytic results, a value of $f_{\text {med }}$ can be chosen, the parton shower can be computed and stopped at the partonic level or evolved using the Lund model to the hadronic level, and then clustered using the anti- $k_{T}$ algorithm and properties like collimation or jet shapes can then be extracted.

In a MC treatment of the shower evolution, using a constant value of $f_{\text {med }}$ to characterize the medium is not needed and in fact not realistic once a comparison with data is desired. Following the procedure in Ref. [45], the value of $f_{\text {med }}$ is determined event by event by embedding the hard process into a hydrodynamical medium [49] starting from a binary vertex which is at $\left(x_{0}, y_{0}\right)$ and following an eikonal trajectory $\zeta$ through the medium evaluating the line integral

$$
f_{\text {med }}=K_{f} \int d \zeta[\epsilon(\zeta)]^{3 / 4}(\cosh \rho(\zeta)-\sinh \rho(\zeta) \cos \psi),
$$

where $\epsilon$ is the local energy density of the hydrodynamical medium, $\rho$ the local flow rapidity and $\psi$ the angle between the flow and the direction of parton propagation. Events are then generated for a large number of random $\left(x_{0}, y_{0}\right)$ sampled from the transverse overlap profile

$$
P\left(x_{0}, y_{0}\right)=\frac{T_{A}\left(\mathbf{r}_{\mathbf{0}}+\mathbf{b} / \mathbf{2}\right) T_{A}\left(\mathbf{r}_{\mathbf{0}}-\mathbf{b} / \mathbf{2}\right)}{T_{A A}(\mathbf{b})},
$$

where $T_{A}$ is a nuclear thickness function $T_{A}(\mathbf{r})=$ $\int d z \rho_{A}(\mathbf{r}, z)$ obtained from the Woods-Saxon density $\rho_{A}(\mathbf{r}, z)$, and all observables are averaged over a sufficiently large number of events. This leaves a single dimensionful parameter $K_{f}$ characterizing the strength of the coupling between parton and medium which is tuned to reproduce the measured nuclear suppression factor $R_{A A}$ in central $200 \mathrm{GeV} \mathrm{AuAu} \mathrm{collisions} \mathrm{(see} \mathrm{Ref.} \mathrm{[45]).}$
In practice, this procedure leads to $\left\langle f_{\text {med }}\right\rangle \approx 0.4$ which we will use in the analytical expressions when a comparison with data is intended.

\section{B. Medium-modified jet energy collimation}

In this section we compare our NLLA (15) and LLA [33] predictions for the energy collimation with YAJEM+BW. The analysis is carried out for gluon and quark jets independently and including all particles in an event, i.e. no detector effects are simulated in this section.

We generate thousands of gluon and quark dijets (i.e. back-to-back jets) for different fixed values of the center-of-mass energy $\sqrt{s}$ taken in the range $\sqrt{s}=$ 100-1200 GeV. By doing so, we fix the energy of the leading initial parton to be $E_{\mathrm{lp}}=\sqrt{s} / 2$ for each member in the dijet. The values of $E_{\mathrm{lp}}$ are thus not selected as in the standard procedure by sampling the initial energy (or $p_{T}$ ) distribution of partons provided by parton distribution functions (PDFs) [50], nuclear parton distribution functions (nPDFs) [51] and the LO matrix elements of the partonic cross section as we do later when comparing with data.

Jets are then reconstructed by using the anti- $k_{T}$ algorithm $[39,40]$ inside the cone of radii $R=1.0,0.3\left(\Theta_{0}\right)$, in agreement with the hard collinear approximation where the NLLA and LLA predictions should be tested. Reconstructed jets can be sorted by energy $\left(E_{\text {jet }, 1}>E_{\text {jet,2 }}>\ldots\right)$ event by event such that the most energetic one $\left(E_{\text {jet, } 1}\right)$ can be randomly selected from its pair $\left(E_{\text {jet,2}}\right)$ for the analysis. We purposely use the default algorithm used by all LHC experiments. Our motivation to do so from this subsection for the energy collimation is based on the fact that we then compare our PYTHIA6 and YAJEM+BW results for the jet shapes with the CMS data in Sec. IV, where jets are reconstructed with the anti- $k_{t}$ algorithm (see Ref. [8]). Besides, the anti- $k_{T}$ is the most robust jet reconstruction algorithm for $p p$ and $\mathrm{PbPb}$ collisions at the LHC with respect to underlying events and pileup.

The jet reconstruction radius coincides with the opening angle of the jet $\Theta_{0}=R$ and $\Theta=r$ with that of the sub-jet. The cone $R$ contains the reconstructed average energy flux $\left(E_{\mathrm{rec}}\right)$ of the jet $A$ and $r$ the energy flux of the sub-jet $B$ $\left(x E_{\text {rec }}\right)$, as illustrated in Fig. 2.

The energy collimation can be then extracted from the angular distribution of the energy flux in $r<R: \frac{1}{N_{\text {jets }}} \frac{d^{2} N}{d e d r}$ for $r<R\left(\Theta<\Theta_{0}\right)$. The recovered jet energy $E_{\text {rec }}$ can be obtained by integrating $\frac{1}{N_{\text {jets }}} \frac{d^{2} N}{d e d r}$ over the whole range $0<r \leq R$ such that the fraction $x$ of the jet energy carried by the sub-jet $r<R$ can be written in the form

$$
\begin{aligned}
x & =\frac{1}{E_{\mathrm{rec}}} \int_{0}^{r} d r^{\prime} \int d e e \frac{d^{2} N}{d e d r^{\prime}}, \\
E_{\mathrm{rec}} & =\int_{0}^{R} d r^{\prime} \int \operatorname{dee} \frac{d^{2} N}{d e d r^{\prime}} .
\end{aligned}
$$




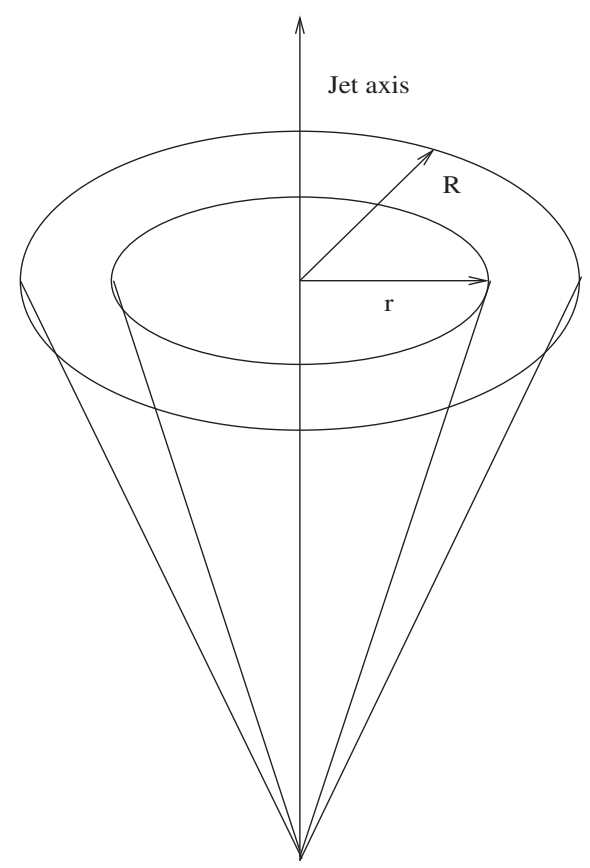

FIG. 2. Jet $\left(\Theta_{0}=R\right)$ and sub-jet $(\Theta=r)$ cones sharing the bulk of the jet energy.

By fixing the energy fraction $x$ in Eq. (21) to be large, $x>0.5$, we can numerically compute the sub-jet radius $r$ where the bulk $x E_{\text {rec }}$ of the reconstructed jet energy is contained. If the same procedure is repeated for different values of the center-of-mass energy $\sqrt{s}$, the energy evolution of the collimation $r\left(E_{\mathrm{rec}}\right)$ can be then displayed as a function of $E_{\text {rec }}$ for a fixed sub-jet energy fraction $x$.

The FASTJET package $[39,40]$ provides the invariant mass $m_{j}=\sqrt{E_{j}^{2}-\vec{p}_{j}^{2}}$ of each jet independently from its pair if jets are sorted by the invariant mass $\left(m_{1}>m_{2}>\ldots\right)$, where $m_{1}$ is the invariant mass of the first jet and $m_{2}$ is the invariant mass of the second jet. The reconstructed virtuality of the jet inside small radii $R \ll 1$ can be related to the invariant mass of the dijet $M_{j j} \gg m_{j}$ through

$$
Q=\frac{M_{j j}}{2} R,
$$

where $M_{j j}$ can be expressed in terms of each $m_{j}$,

$$
\begin{aligned}
M_{j j}^{2}= & m_{1}^{2}+m_{2}^{2}+2 E_{1} E_{2} \\
& -2 E_{1} E_{2} \sqrt{\left(1-\frac{m_{1}^{2}}{E_{1}^{2}}\right)\left(1-\frac{m_{2}^{2}}{E_{2}^{2}}\right)} \cos \phi,
\end{aligned}
$$

which should be evaluated with $m_{1} \neq m_{2}, E_{1} \neq E_{2}$ and $\phi \approx \pi$ for clustered back-to-back jets. Indeed, the jet finder misses part of the initial jet energy $E_{\mathrm{lp}}$ and invariant mass $m_{\mathrm{lp}}$ such that, the new values inside $R$ are biased to smaller ones. Therefore, the reconstructed invariant mass of the dijet $M_{j j}$ should be estimated as given in Eq. (23) for the biased $E_{i}$ and $m_{i}$ obtained with FASTJET. The result in Eq. (22) can be checked to be in good agreement with the averaged one $Q=E_{\mathrm{rec}} R$ displayed in Tables II and III in Secs. III C and III D, respectively; the smaller the $R$ values, the more important the bias and the better the agreement in the collinear limit, provided $R \geq \Lambda_{\mathrm{QCD}} / E_{\mathrm{rec}}$. We rewrite Eq. (17) in the form,

$$
\begin{aligned}
r_{A} & =R\left(\frac{Q}{\Lambda_{\mathrm{QCD}}}\right)^{-\gamma_{A}\left(x, N_{s}\right)}, \quad C_{g}=N_{c}=3, \\
C_{q} & =C_{F}=\frac{4}{3},
\end{aligned}
$$

for the phenomenological treatment of the gluon $\left(C_{g}=N_{c}\right)$ and quark $\left(C_{q}=C_{F}\right)$ jets with $r_{A} \leq R$. We choose $\Lambda_{\mathrm{QCD}}=300 \mathrm{MeV}$, the same value as in the PYSHOW showering algorithm and do not use the Lund model for the hadronization of partons into hadrons in this section $[13,14]$. The result for the energy collimation extracted from YAJEM+BW (21) will be compared with the mediummodified NLLA and LLA formulas (15). The medium modification parameter $f_{\text {med }}$ is set to its mean value $\langle f\rangle_{\text {med }}=0.4$, obtained from averaging over the hydrodynamical model. The result of the numerical inversion of Eqs. (15) and (16) will be displayed in the form given by Eq. (24) in both cases.

\section{Medium-modified jet energy collimation in gluon jets}

In Table II, we display gluon dijets at three different center-of-mass energies $\sqrt{s}=150-500 \mathrm{GeV}$, which are reconstructed by using the anti- $k_{T}$ algorithm $[39,40]$ for the radii $R=1$ and $R=0.3$. As described above, $E_{\text {rec }}$ is the recovered jet energy of the leading parton $E_{\mathrm{rec}}=\hat{z} \sqrt{s} / 2$ inside the cone $R$ and $Q$ is the jet virtuality. Note that the recovered energy of the leading parton equals the jet energy

TABLE II. Reconstructed jet energies inside the cone radii $R=1.0$ and $R=0.3$.

\begin{tabular}{lrrrrrrr}
\hline \hline$\sqrt{s}(\mathrm{GeV})$ & $R$ & $E_{\mathrm{rec}}(\mathrm{GeV})$ & $Q(\mathrm{GeV})$ & $R$ & $E_{\mathrm{rec}}(\mathrm{GeV})$ & $Q(\mathrm{GeV})$ \\
\hline 150 & 1.0 & 64.8 & 64.8 & 0.3 & 46.3 & 13.8 \\
300 & 1.0 & 131.3 & 131.3 & 0.3 & 98.0 & 29.4 \\
500 & 1.0 & 220.4 & 220.4 & 0.3 & 168.9 & 50.7 \\
\hline \hline
\end{tabular}

TABLE III. Reconstructed jet energies inside the cone radii $R=1.0$ and $R=0.3$.

\begin{tabular}{lrrrrrr}
\hline \hline$\sqrt{s}(\mathrm{GeV})$ & $R$ & $E_{\mathrm{rec}}(\mathrm{GeV})$ & $Q(\mathrm{GeV})$ & $R$ & $E_{\mathrm{rec}}(\mathrm{GeV})$ & $Q(\mathrm{GeV})$ \\
\hline 150 & 1.0 & 70.0 & 70.0 & 0.3 & 58.5 & 17.6 \\
300 & 1.0 & 141.0 & 141.0 & 0.3 & 100.1 & 30.0 \\
500 & 1.0 & 236.0 & 236.0 & 0.3 & 205.9 & 61.5 \\
\hline \hline
\end{tabular}


inside $R$ after reconstruction. We can see that gluon jets carry the energy fractions $\hat{z} \sim 4 / 5$ and $2 / 3$ of the leading parton for $R=1.0$ and $R=0.3$, respectively. Indeed, $\sqrt{s} / 2$ is the energy of the leading parton spread in the whole hemisphere and $\sqrt{s} / 2-E_{\text {rec }}$ is that part of the jet energy that is lost outside $R$, which should not be confused with the jet energy inside the cone shell $r \leq r^{\prime} \leq R$. We can see that the correlation between parton kinematics and reconstructed jet kinematics gets increasingly blurred for small reconstruction radii. Thus, the choice $R=0.3$ used by the CMS experiment provides a more severely biased jet energy.

In Fig. 3, we display the energy collimation at $R=1.0$ in gluon jets,

$$
r_{g}=R\left(\frac{Q}{\Lambda_{\mathrm{QCD}}}\right)^{-\gamma_{g}\left(x, N_{s}\right)}
$$

in the energy range $60 \leq E_{\mathrm{rec}}(\mathrm{GeV}) \leq 600$ for RHIC and LHC phenomenology. We choose the energy fractions $x=0.5, x=0.8$ and compare the NLLA prediction (15) with the LLA (16) and YAJEM+BW (21) predictions for $\langle f\rangle_{\text {med }}=0.4$. The disagreement between the LLA prediction and YAJEM+BW is quite substantial and is mainly due to the lack of other perturbative contributions in this calculation, whereas the NLLA prediction improves the agreement. As expected for $x=0.5$, the $\mathcal{O}\left(\alpha_{s}\right)$ correction in the NLLA formula (15) proves to be larger than for $x=0.8$. The shape of the energy collimation provided by the NLLA (15) and the LLA (16) are identical but steeper than the slope of the energy collimation provided by YAJEM+BW. Therefore, the NLLA and LLA predictions overestimate the energy collimation compared to the YAJEM+BW prescription.

Decreasing the jet radius to the value used by the CMS experiment at $2.76 \mathrm{TeV} \mathrm{PbPb}$ collisions, $R=0.3$, leads to a sizable hardening of the biased jet which may provide a better comparison between the NLLA, LLA and YAJEM+BW predictions for the jet energy collimation. The bias drives results to a generic outcome, so differences in the comparison must disappear as the bias gets stronger. That is why, in Fig. 4 we display the same curves as in Fig. 3 for $R=0.3$.
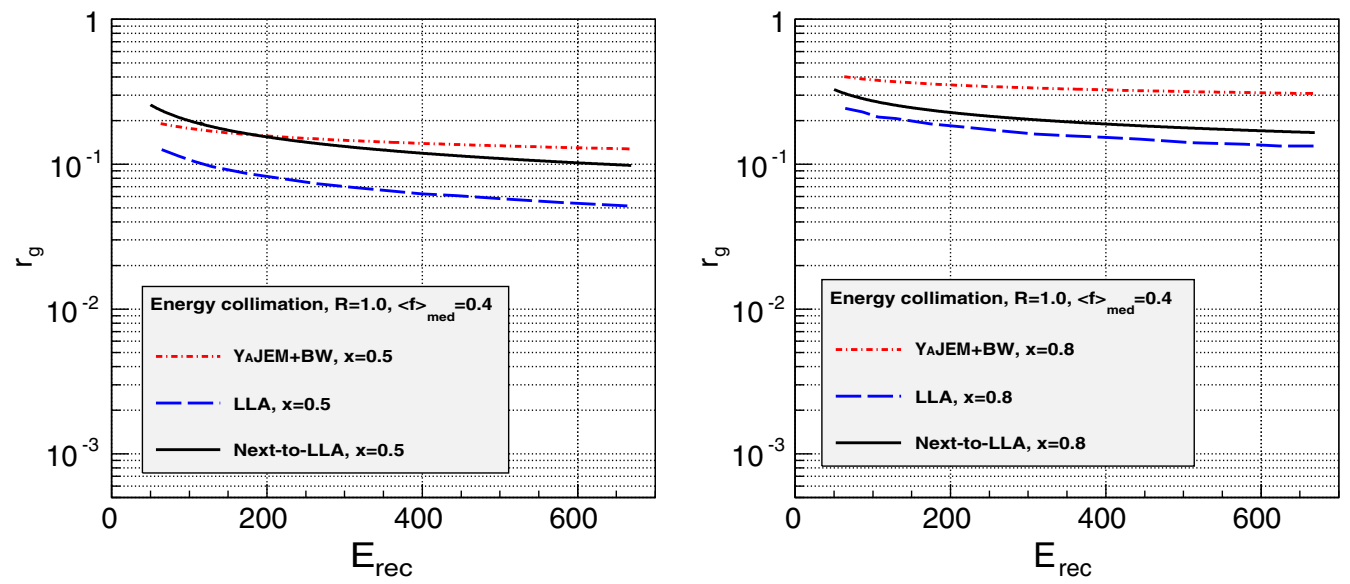

FIG. 3 (color online). Energy collimation inside a gluon jet for $x=0.5$ (left) and $x=0.8$ (right) with $R=1.0$.
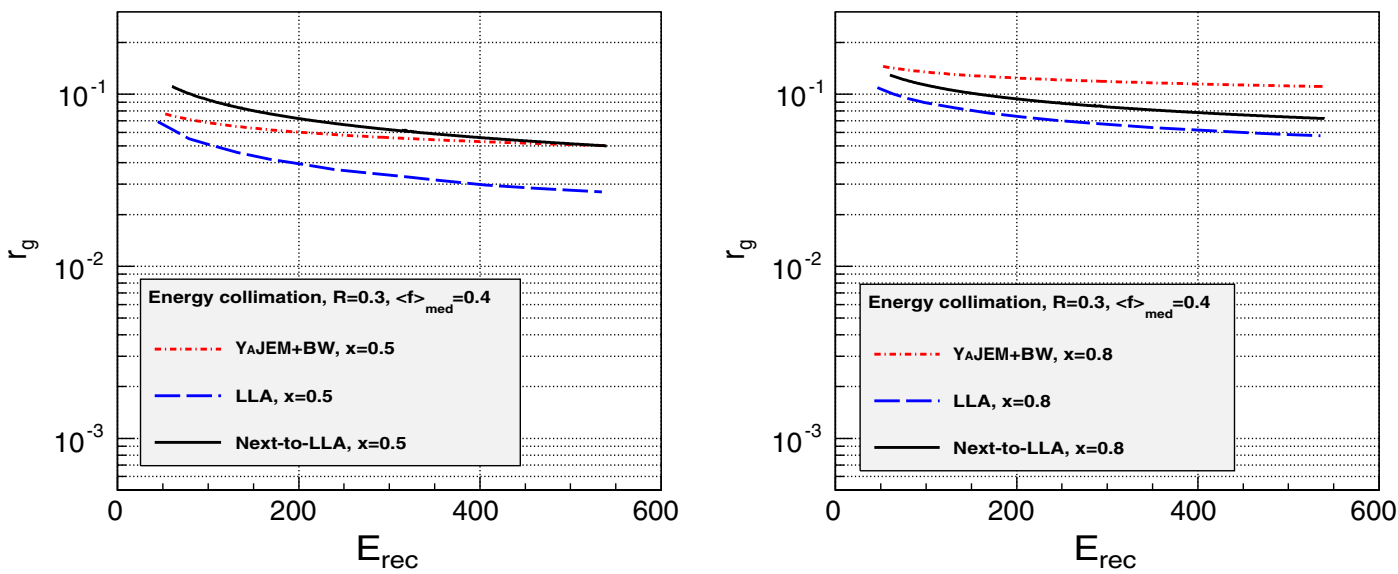

FIG. 4 (color online). Collimation of energy inside a gluon jet for $x=0.5$ (left) and $x=0.8$ (right) with $R=0.3$. 
We can see that the description provided by the NLLA (21), LLA (16) and YAJEM+BW (21) calculations are in better agreement with one another than the results displayed in Fig. 3 for $R=1.0$. As expected, in the LLA, NLLA and YAJEM+BW computations, the energy collimation is stronger as the jet energy increases.

As a consequence of jet quenching in high-energy heavyion collisions, medium-modified showers are expected to broaden compared with vacuum showers. This effect can be quantified via the shower energy collimation by taking the ratios $r_{g \text {,med }} / r_{g \text {,vac }}$ with $\langle f\rangle_{\text {med }}=0.4$ for the medium in the numerator and $\langle f\rangle_{\text {med }}=0$ for vacuum in the denominator with the NLLA formula (15) and the YAJEM analysis (21). The ratios are displayed in Fig. 5 for the energy fractions $x=0.5,0.8,0.9$ and the jet radius $R=0.3$. For $x=0.5$, the NLLA formula predicts a sub-jet broadening that is twice as large (i.e. smaller energy collimation) than that in YAJEM $+\mathrm{BW}$, while for $x=0.8$ to $x=0.9$ the agreement is improved but still different by a factor of $\sim 1.5-1.2$, reaching the best agreement for $x=0.9$. Thus, as the energy fraction $x$ increases, the jet broadening inside the smaller cone $r$ decreases. As expected, the NLLA correction seems to play a more important role as $x$ decreases. The latter can be observed in Fig. 5 as one compares the shapes of the NLLA prediction with YAJEM+BW. The YAJEM+BW prediction tends to flatten while the NLLA formula increases, making the vertical difference higher as the energy scale increases.

\section{Medium-modified jet energy collimation in quark jets}

In Table III, we display quark dijets for the same values of the center-of-mass energy and $R$. We can see that the recovered jet energy slightly increases compared with that displayed in Table II for gluon jets. The energy collimation inside quark jets (17) can be rewritten in the form,

$$
r_{q}=R\left(\frac{Q}{\Lambda_{\mathrm{QCD}}}\right)^{-\gamma_{q}\left(x, N_{s}\right)}
$$

Accordingly, in Fig. 6 and Fig. 7, we display the quark jet energy collimation for the energy fractions $x=0.5$ and
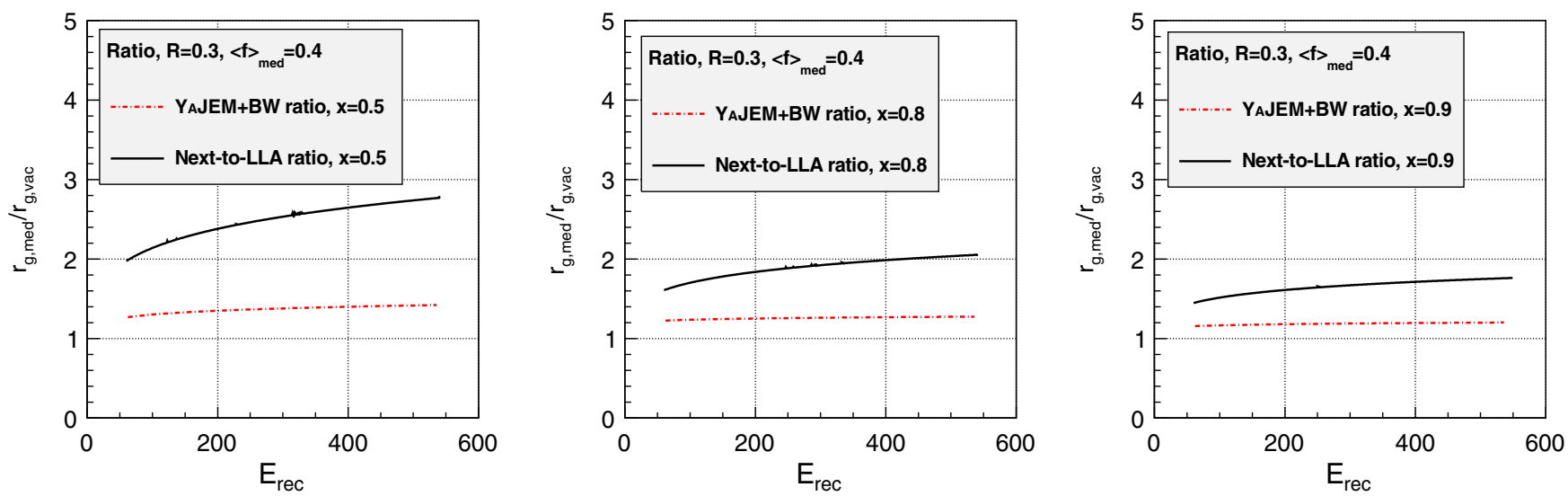

FIG. 5 (color online). Medium-modified and vacuum energy collimation ratios $r_{g \text {,med }} / r_{g, \text { vac }}$ for $x=0.5$ (left), $x=0.8$ (center) and $x=0.9$ (right) with $R=0.3$.
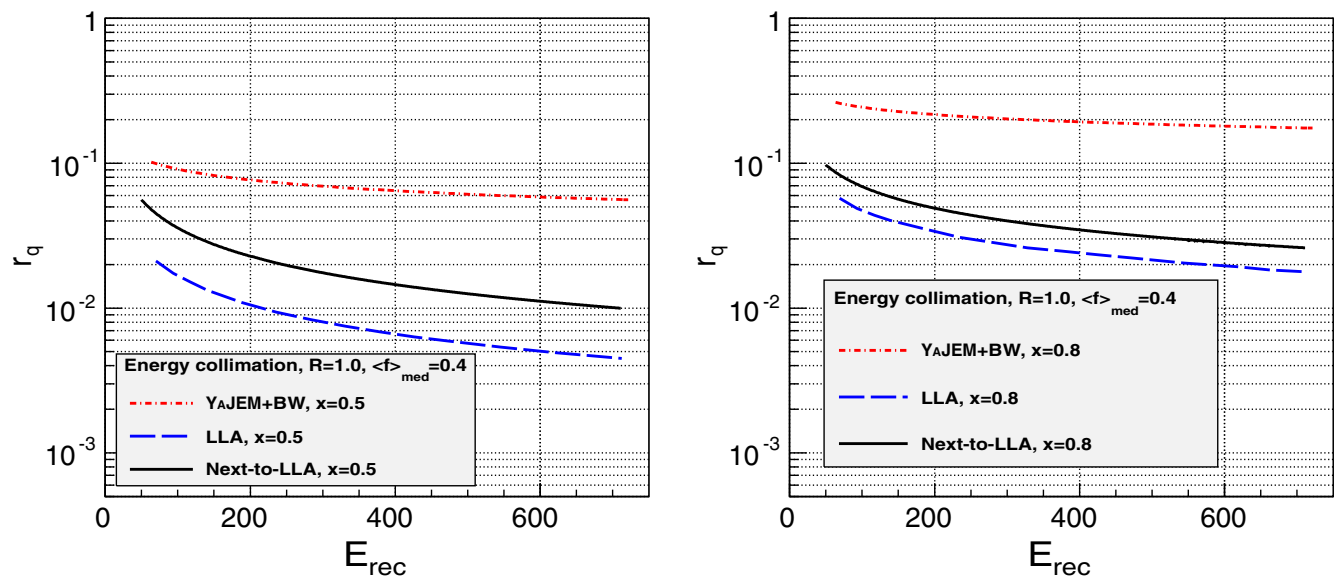

FIG. 6 (color online). Collimation of energy inside a gluon jet for $x=0.5$ (left) and $x=0.8$ (right) with $R=1.0$. 

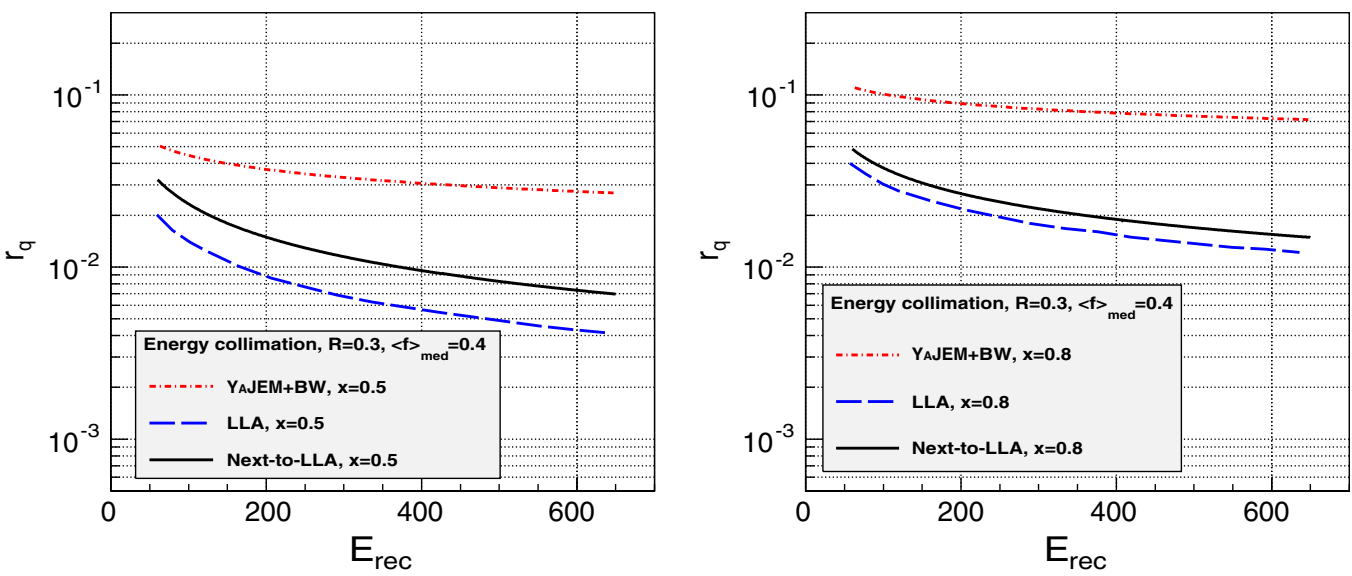

FIG. 7 (color online). Collimation of energy inside a quark jet for $x=0.4$ (left) and $x=0.8$ (right) with $R=0.3$.

$x=0.8$ by the sub-jet with the medium modification value $\langle f\rangle_{\text {med }}=0.4$.

As for gluon jets, our predictions are in better agreement with YAJEM+BW for $R=0.3$ than for $R=1.0$. For $R=1.0$, the NLLA predictions underestimate YAJEM+BW for $x=0.5$ and $x=0.8$. However, for $R=0.3$, the disagreement is reduced as for gluon jets. Furthermore, the correction due to the shift in $\ln x$ is smaller in a quark jet compared to a gluon jet and LLA predictions in gluon jets are in better agreement with YAJEM+BW than in quark jets. The last statements suggest that NLLA and LLA predictions should be in better agreement with YAJEM+BW for much harder jets, i.e. $R=0.1$ as displayed in Fig. 8. The study of smaller jet resolutions such as $R=0.1$ which further biases QCD showers, is shown to always improve quark/gluon tagging at the LHC [52]. In Fig. 9, we display the ratios $r_{q \text {,med }} / r_{q \text {,vac }}$ of the energy collimation in the medium and the vacuum. The results clearly show the quark jet broadening as a consequence of jet quenching. The comparison between the NLLA and LLA predictions is worse than for gluon jets. In Fig. 10, we compare the LLA, NLLA and PYTHIA energy collimation in the vacuum.
The disagreement between the LLA, NLLA and PYTHIA6 predictions is more pronounced in the vacuum, which further explains the huge difference displayed by the ratios in Fig. 5 and Fig. 9.

In Table IV, we present the values of the slopes $\gamma_{A}\left(x, N_{S}\right)$ provided by YAJEM+BW $\left(\langle f\rangle_{\text {med }}=1.4\right)$ and PYTHIA6. As displayed in the above figures for the energy collimation, the values are smaller than in the NLLA and LLA calculations presented in Table I. However, the trends shown by the variation of $\gamma_{A}\left(x, N_{s}\right)$ as a function of $x$ and $N_{s}$ are similar $\left(\gamma_{q}>\gamma_{g}\right)$. In particular, the slopes decrease as the energy fraction decreases for a given value of $N_{s}$. For a fixed value of $x$, the energy collimation flattens as $N_{s}$ increases. We can see that our calculations and YAJEM+BW predict a much stronger energy collimation in quark jets than in gluon jets. Physically, this is because gluon jets have a color charge roughly twice as large $\left(N_{c} / C_{F}=9 / 4\right)$ than quark jets, or equivalently, gluon jet multiplicities are higher than quark jet multiplicities by the same factor asymptotically [53]. Moreover, the first splitting dominates the jet width, which for quark jets only has the available splitting $q \rightarrow q g$ where the emitted gluon is
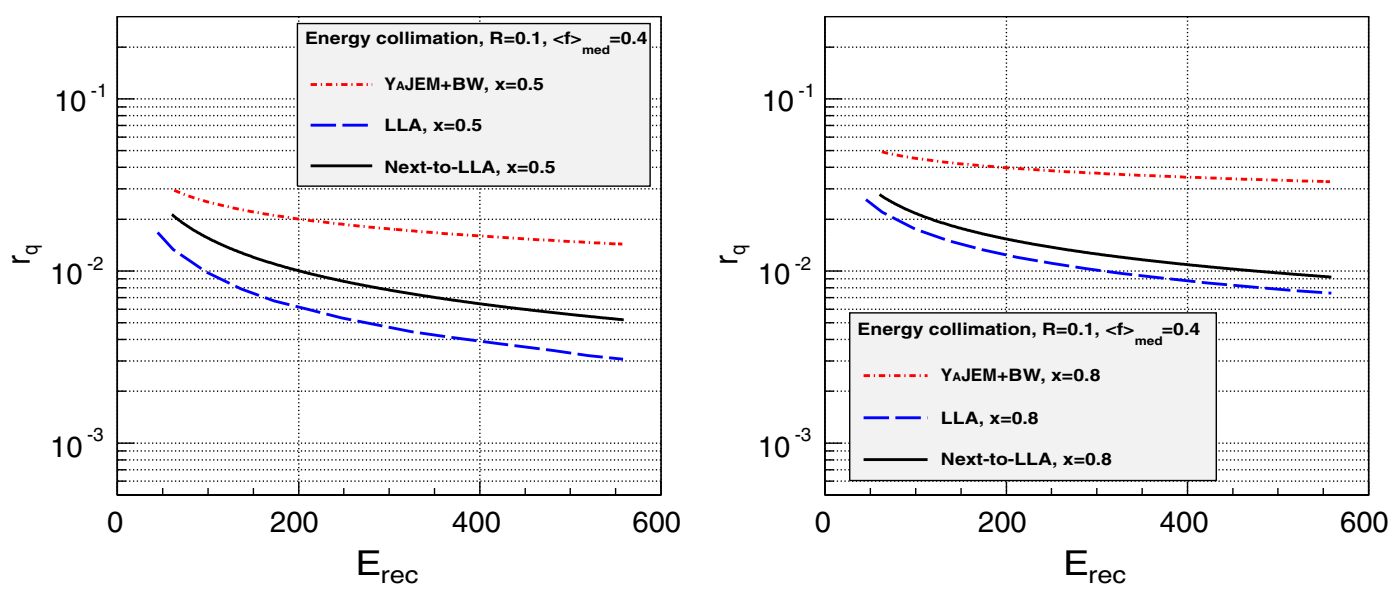

FIG. 8 (color online). Collimation of energy inside a gluon jet for $x=0.5$ (left) and $x=0.8$ (right) with $R=0.1$. 

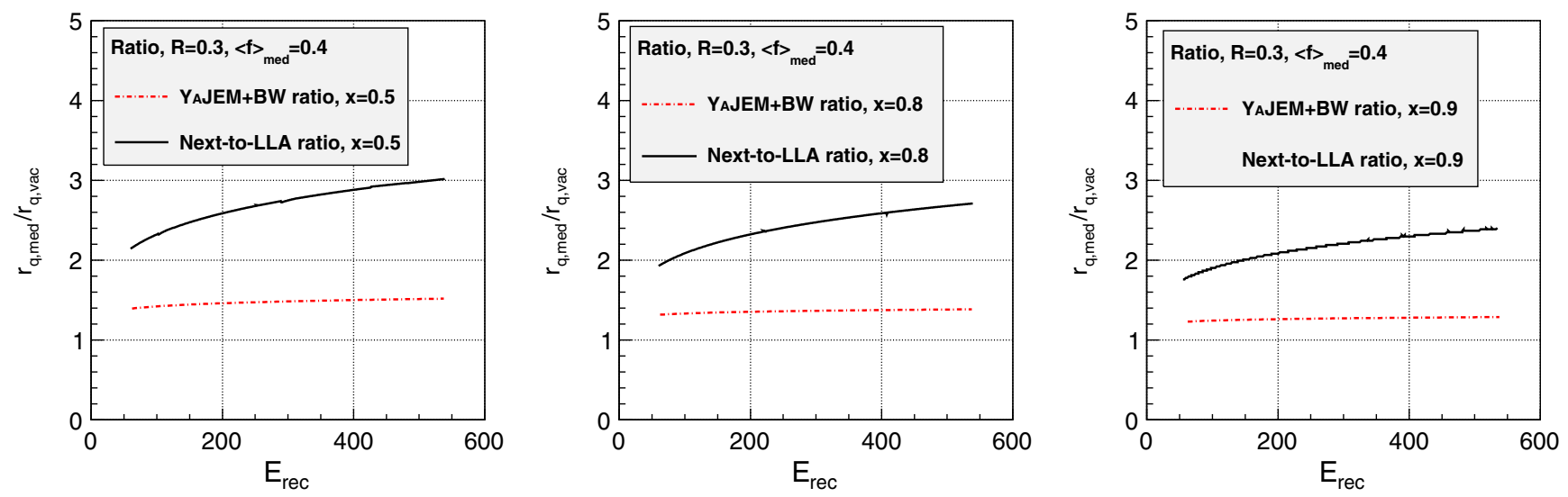

FIG. 9 (color online). Medium-modified and vacuum energy collimation ratios $r_{\mathrm{q}, \text { med }} / r_{\mathrm{q} \text {, vac }}$ for $x=0.5$ (left), $x=0.8$ (center) and $x=0.9$ (right) with $R=0.3$.
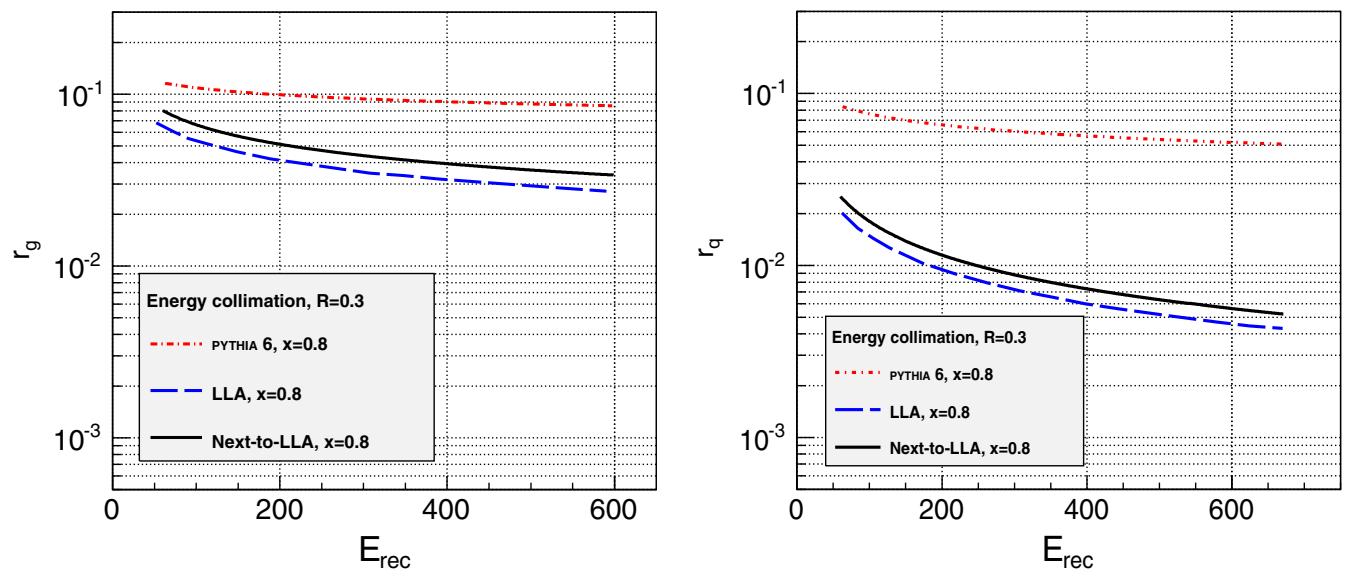

FIG. 10 (color online). Collimation of energy inside a gluon jet (left) and quark jet (right) for $x=0.8$ with $R=0.3$ in the vacuum $\left(N_{s}=1\right)$.

preferentially soft and does not alter the transverse jet shape, whereas gluon jets can split into $g \rightarrow q \bar{q}$ pairs where both quarks tend to be equally hard, which can widen the shape substantially. In both YAJEM+BW and the calculation, the energy collimation is hence steeper for quark jets than for gluon jets.

Equations (15) and (16) provide a simple description of the jet energy collimation under consideration and cannot be in perfect agreement with the YAJEM+BW description. This last point suggests that other perturbative contributions arising from the splittings $q \rightarrow q g$ and $g \rightarrow q \bar{q}$ should be included in Eq. (1) in the form $D_{q}\left(x, E \Theta_{0}, x E \Theta\right)=$ $D_{q}^{q}\left(x, E \Theta_{0}, x E \Theta\right)+D_{q}^{g}\left(x, E \Theta_{0}, x E \Theta\right)$ for quark jets and

TABLE IV. YAJEM+BW values of the slope $\gamma_{A}\left(x, N_{s}\right)$ of the energy collimation for $N_{s}=1.4$ (medium) and PYTHIA6 values for $N_{s}=1$ (vacuum).

\begin{tabular}{lccccc}
\hline \hline YAJEM+BW & $x=0.5$ & $x=0.8$ & PYTHIA6 & $x=0.5$ & $x=0.8$ \\
\hline$\gamma_{g}(x, 1.4)$ & 0.17 & 0.11 & $\gamma_{g}(x, 1)$ & 0.21 & 0.14 \\
$\gamma_{q}(x, 1.4)$ & 0.24 & 0.17 & $\gamma_{q}(x, 1)$ & 0.29 & 0.20 \\
\hline \hline
\end{tabular}

$D_{g}\left(x, E \Theta_{0}, x E \Theta\right)=D_{g}^{g}\left(x, E \Theta_{0}, x E \Theta\right)+D_{g}^{q}\left(x, E \Theta_{0}, x E \Theta\right)$ for gluon jets, with the full resummed contribution of the soft-collinear logarithms in DGLAP FFs [30,43]. Indeed, as the jet energy increases, the contributions from the double logarithmic contributions $\alpha_{s} \frac{d z}{z} \frac{d \Theta}{\Theta}\left(z=E_{g} / E\right)$ increase asymptotically, which may explain why the difference between YAJEM+BW and the NLLA predictions gets wider as the jet energy $E$ increases. Moreover, the more accurate treatment of phase space in both PYTHIA and YAJEM have not been taken into account in the NLLA and LLA calculations.

\section{E. Hadronization effects in the energy collimation}

In Fig. 11 we display the energy collimation inside gluon and quark jets in the vacuum using PYTHIA6. The role of hadronization is displayed by comparing the energy collimation for final-state hadrons and final-state partons clustered inside the radius $R=0.3$ in the energy range $50 \leq E_{\text {rec }}(\mathrm{GeV}) \leq 700$ by using the anti- $k_{T}$ algorithm $[39,40]$. The hadronic energy collimation has been labeled as "PYTHIA6" and the partonic energy collimation is labeled 

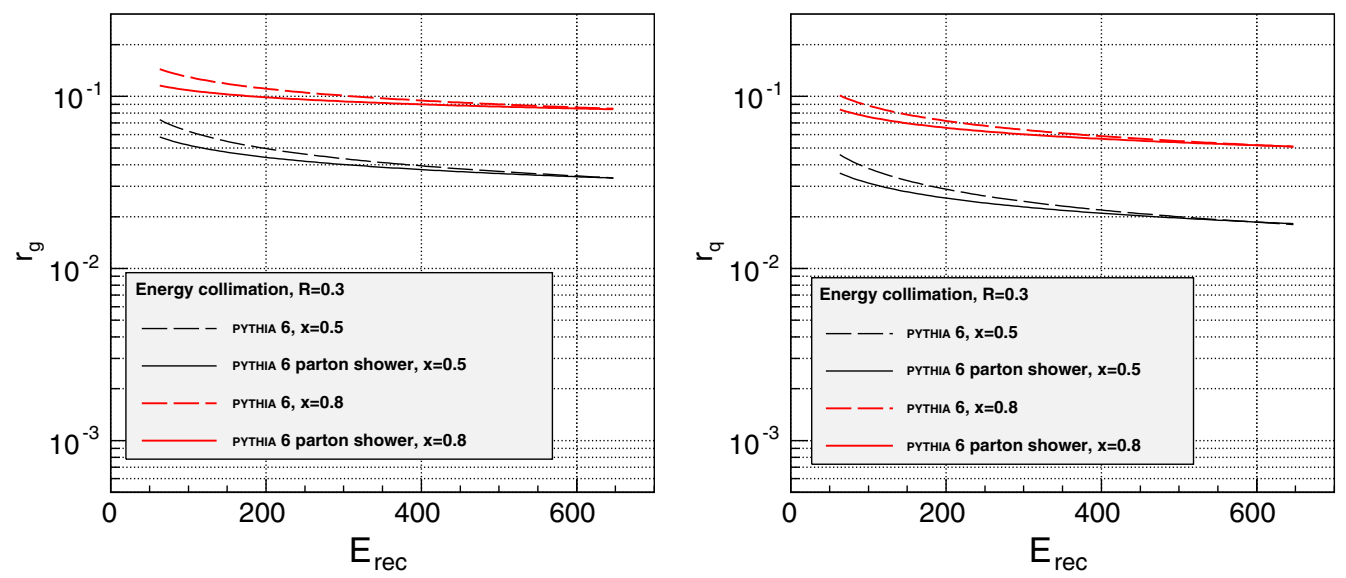

FIG. 11 (color online). Parton versus hadron energy collimation for $x=0.5$ and $x=0.8$ inside a gluon jet (left) and a quark jet (right) with $R=0.3$.

as "PYTHIA6 parton shower." Since the hadronization is modeled to occur outside the medium, we limited the comparison to PYTHIA6 since the results would be identical for YAJEM+BW with a slightly larger normalization (see Figs. 4 and 7 for comparison) as a consequence of the jet broadening. As we can see, the hadronization biases the partonic energy collimation for jet energies $<400 \mathrm{GeV}$ but this effect is $\sim 5 \%$ at RHIC energy scales and smaller than $1 \%$ at LHC energy scales. For jet energies $>400 \mathrm{GeV}$ the role of hadronization becomes negligible and therefore irrelevant for the study of this observable. In general hadronic showers are less collimated than a fictitious parton shower at small energy scales.

\section{JET SHAPE: COMPARISON WITH PbPb CMS DATA}

The integrated jet shape $\Psi(r ; R)$ measures the fraction of the jet energy of size $R$ contained in a sub-cone of size $r$ such that $\Psi(R ; R)=1$. The differential jet shape reads [36],

$$
r \psi(r ; R)=r \frac{d \Psi}{d r},
$$

where

$$
\begin{aligned}
\frac{d \Psi}{d r} & =\frac{1}{E_{\mathrm{rec}}} \int d e e \frac{d^{2} N}{d e d r^{\prime}}, \\
E_{\mathrm{rec}} & =\int_{0}^{R} d r^{\prime} \int \operatorname{dee} \frac{d^{2} N}{d e d r^{\prime}} .
\end{aligned}
$$

Hence, the integration of Eq. (27) leads to the expression written in Eq. (21) for the energy fraction $x$ used in the framework of the energy collimation, which will be identified with the integrated jet shape hereafter: $x \equiv \Psi(r ; R)$. Our NLLA and LLA predictions for the integrated jet shape will be therefore based on the maximal angular aperture $\Theta \equiv r$ where the bulk of the jet energy is contained, as we discussed in Sec. II A. For the first time, in this paper the jet shape is computed from the jet energy collimation within the same NLLA and LLA schemes.

First of all, we describe how the Monte Carlo simulation from PYTHIA6 and YAJEM is performed in view of further comparison with LLA, NLLA predictions and CMS data hereafter.

For the computation of the integrated jet shapes extracted from Eq. (27), we will limit our study to charged particles only, as in the CMS experiment [8]. The initial distribution of gluon- and quark-initiated showers for the analysis is determined by the convolution of PDFs and nPDFs with the LO matrix elements of the final cross section at the given hard factorization scale of the process. The LO matrix elements of the partonic cross section can be computed analytically [54]. PDFs and nPDFs are provided by the CTEQ [50] and EKS [51] families for $p p$ and $\mathrm{PbPb}$ collisions in the vacuum and the medium, respectively. The analysis carried out for the jet shapes is hence different than the analysis for the energy collimation in Secs. III C and III D where the center-of-mass energy of the hard parton system was fixed to a certain value $\sqrt{s}$.

A large number of quark and gluon dijets are randomly generated based on the perturbative QCD spectrum inside the energy range $200 \leq \sqrt{s}(\mathrm{GeV}) \leq 600$ and clustered by using the anti- $k_{t}$ algorithm with $R=0.3$. After clustering all charged hadrons with $e>1 \mathrm{GeV}$ inside the given cone $R=0.3$, jet energies $E_{\text {rec }}$ are required to fulfill CMS trigger conditions imposed by the restriction $E_{\text {rec,jet }} \geq 100 \mathrm{GeV}$. The requirement imposed by the trigger selection in the analysis will be referred to as a biased shower, while that including all clustered jet energies will be referred to as an unbiased shower in the following. Accordingly, the fraction of gluon jets in one sample is biased by the trigger condition from $f_{g}^{\mathrm{vac}} \approx 0.4$ in the unbiased case to $f_{g}^{\mathrm{vac}} \approx 0.2$ in the biased case in vacuum showers, and from $f_{g}^{\text {med }} \approx 0.3$ to $f_{g}^{\text {med }} \approx 0.1$ in medium showers. Thus, quark jets are dominant in the analysis, particularly in the medium. 
The fraction of gluon jets in a sample is used for the computation of the mixed integrated jet shape given by the linear combination for gluon and quark jets in the form

$$
\Psi_{\text {mixed }}(r ; R)=f_{g} \Psi_{g}(r ; R)+\left(1-f_{g}\right) \Psi_{q}(r ; R)
$$

for a direct comparison of the LLA (16), the NLLA (15), PYTHIA6 and YAJEM+BW with CMS data.

In YAJEM+BW, $f_{\text {med }}$ is computed event by event as described in Sec. III A. Instead of treating quark and gluon jets independently in this framework, it is more straightforward to mix the differential distributions for the energy flux (27) and compare them with the mixed jet shape from PYTHIA and YAJEM+BW with account of hadronization. By doing so, after averaging over a large number of events, all jets cluster to the biased mean jet-energy value $E_{\text {rec }} \sim$ $140 \mathrm{GeV}$ and the mean medium parameter $\langle f\rangle_{\text {med }} \sim 0.4$.

In order to compare the PYTHIA6 and YAJEM+BW calculations with the LLA and NLLA gluon and quark jet shapes, we solve the equations (15) and (16) numerically $\left[x_{g} \equiv \Psi_{g}(r ; R)\right.$ and $\left.x_{q} \equiv \Psi_{q}(r ; R)\right]$ as a function of $r$ in the interval $0 \leq r \leq R$ in the framework of LLA (NLLA) DGLAP evolution at large $x$, for the first time in this paper. For the computation, we choose the mean jet-energy value $E_{\text {rec }}=140 \mathrm{GeV}$ extracted from PYTHIA6 and YAJEM+BW. Taking the same values for the fraction of gluon jets in a sample $f_{g}^{\text {vac }} \approx 0.2$ in the vacuum $\left(\langle f\rangle_{\text {med }}=0\right)$ and $f_{g}^{\text {vac }} \approx 0.1$ in the medium $\left(\langle f\rangle_{\text {med }}=0.4\right)$, we can evaluate the mixed LLA and NLLA jet shapes (28) equivalently as in the Monte Carlo event generators.

In Fig. 12, we show the LLA, PYTHIA6 and YAJEM+BW predictions for $\langle f\rangle_{\operatorname{med}}=0.4$ jet shapes compared with $p p$ and $\mathrm{PbPb} \mathrm{CMS}$ data in the left panel, and the NLLA, PYTHIA6 and YAJEM+BW jet shapes for $\langle f\rangle_{\text {med }}=0.4$ compared with $p p$ and $\mathrm{PbPb} \mathrm{CMS}$ data in the right panel. The jet shape is displayed in the interval $0.6 \leq$ $x \equiv \Psi(r ; R) \leq 1$ in agreement with the LLA (and NLLA) DGLAP large sub-jet energy fraction $x$ approximation where these calculations are performed. We can see that the LLA and NLLA qualitatively describe the features of the jet shapes in both vacuum and medium but an important disagreement persists. Compared to the LLA calculation, the NLLA calculation approaches the data as the jet shape decreases and particularly for more collimated sub-jets $r$, as expected. Translating this statement to the energy collimation, we show the NLLA correction to widen the energy dependence of $r$ and to increase the difference with the LLA calculation as the subjet energy fraction (jet shape) decreases. Thus, although the NLLA and LLA predictions seem to capture the main ingredients of the hard fragmentation process in this framework, the account of all fragmentation probabilities, mainly those containing soft gluon contributions should be taken into account in a more accurate theoretical framework. PYTHIA6 provides instead a good agreement with $p p$ CMS data for biased jets. YAJEM+BW describes the shower medium modifications by reproducing the jet broadening at slightly larger values of $r$ to the right, similar to the medium-modified NLLA and LLA jet shapes. Though the YAJEM+BW calculation does not reproduce the data points exactly, the curve fits the systematic error bars of the $\mathrm{CMS} \mathrm{PbPb}$ data. As observed, the sub-jet broadening shown by the data is very small but in better agreement with the sub-jet broadening shown by the Monte Carlo simulations than with that shown by the theoretical calculations with the BW prescription.

In Fig. 13 we compare the biased $\left(E_{\text {rec,jet }} \geq 100 \mathrm{GeV}\right)$ and unbiased (all jets) cases obtained with PYTHIA6 and YAJEM+BW with $p p$ (left panel) and $\mathrm{PbPb}$ (right panel) CMS data. The unbiased mean jet energy turns out to be $E_{\text {jet }} \sim 90 \mathrm{GeV}$ after all clustered jets are considered in the analysis without any further trigger bias. Furthermore, the evaluation of the unbiased case through Eq. (28) requires the unbiased gluon jet fractions $f_{g}^{\mathrm{vac}} \approx 0.4$ and $f_{g}^{\text {med }} \approx 0.3$ in the vacuum and in the medium, respectively, as performed here. As can be seen, the shower structure is affected by

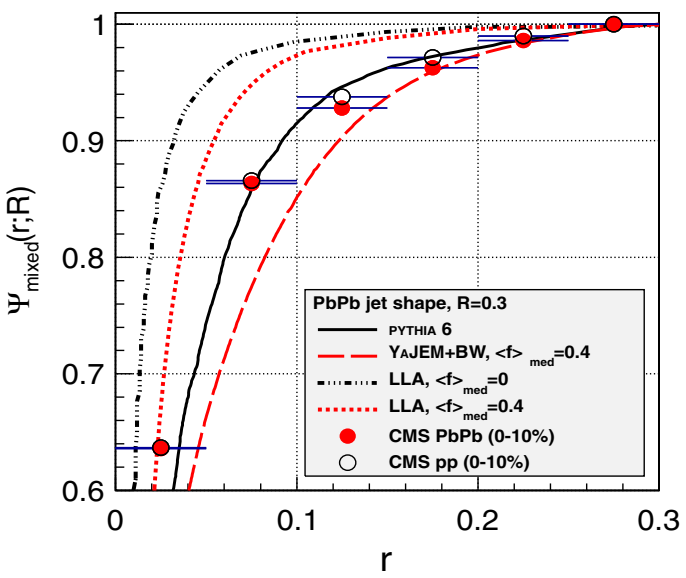

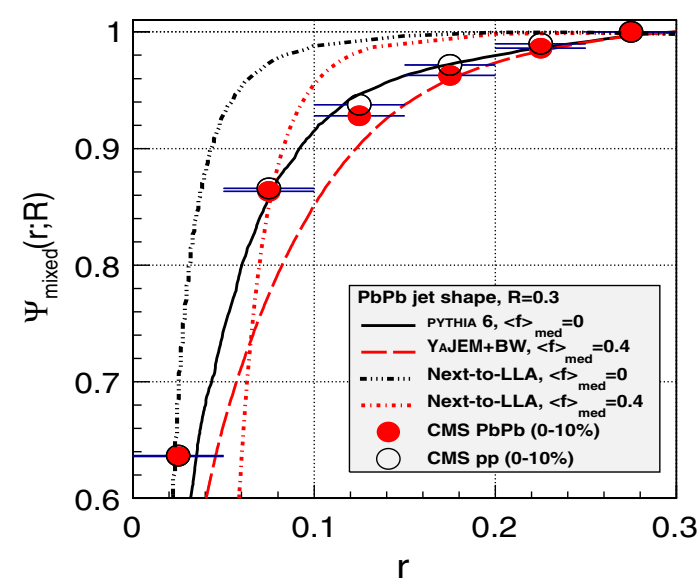

FIG. 12 (color online). Jet shape for CMS $p p$ and $\mathrm{PbPb}$ data with $R=0.3$, compared with PYTHIA6, YAJEM+BW, the LLA formula (left panel) and the NLLA formula (right panel). 

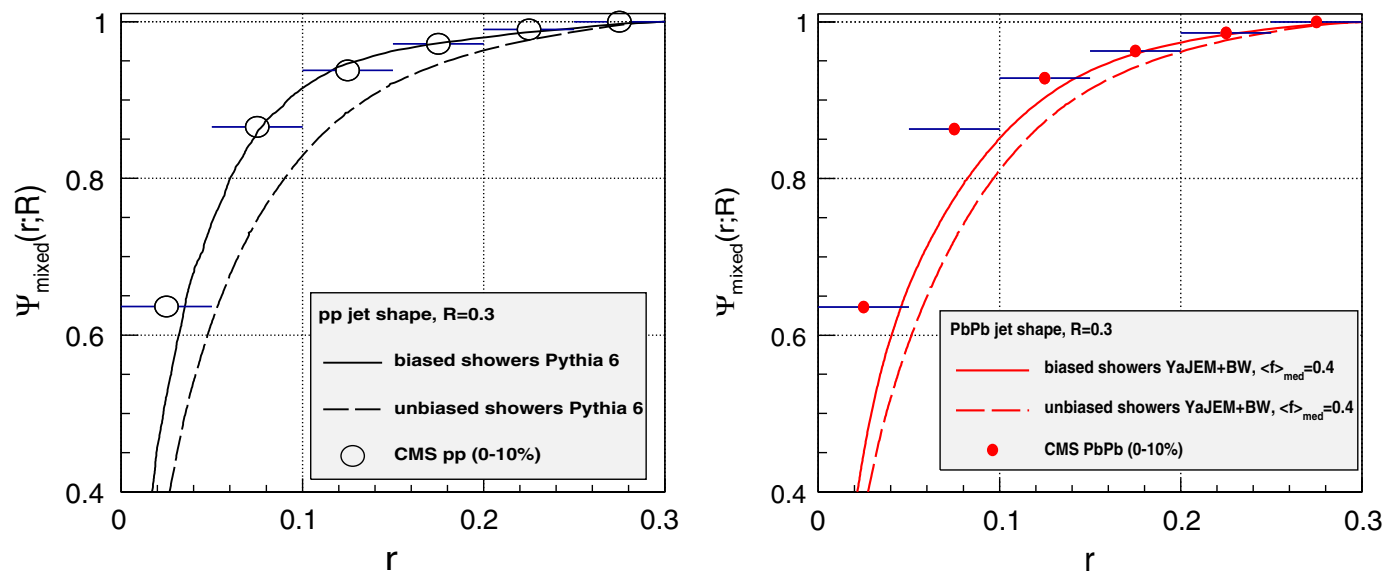

FIG. 13 (color online). Biased versus unbiased jet shape for CMS $p p$ data (left panel) and $\mathrm{PbPb}$ data (right panel) with $R=0.3$.
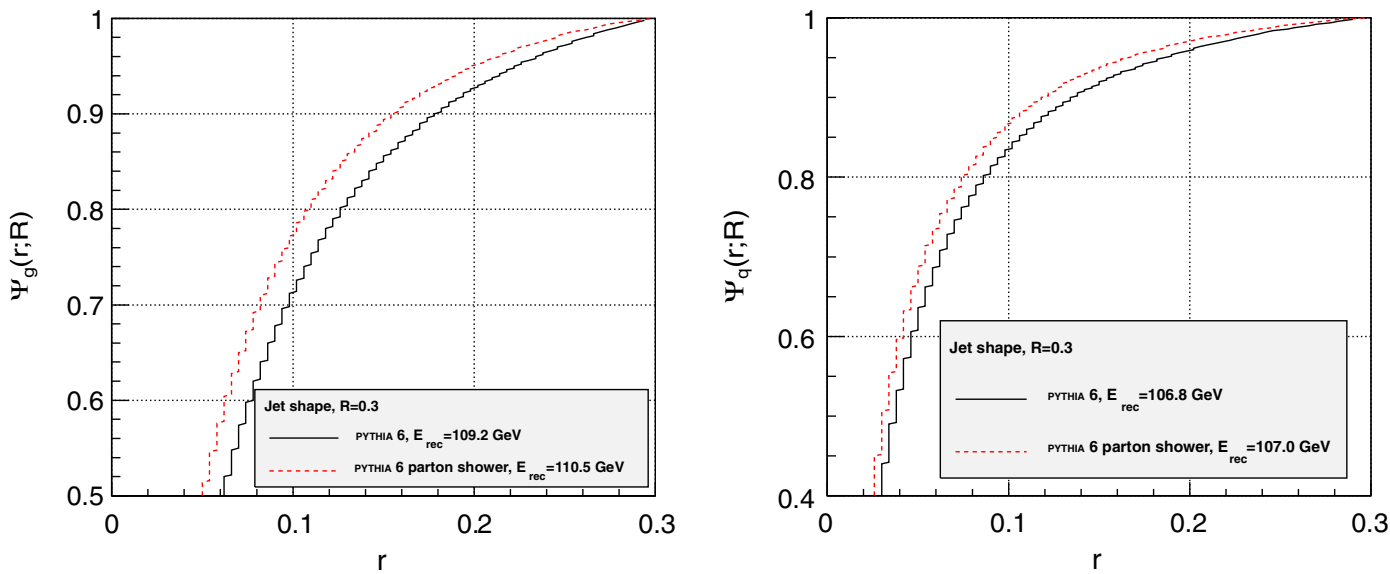

FIG. 14 (color online). Parton versus hadron jet shape inside a gluon jet (left) and a quark jet (right) with $R=0.3$.

imposing a jet-energy condition which leads to a better agreement between the biased jet shape and the data than the unbiased jet shape.

\section{A. Hadronization effects in gluon and quark jet shapes}

Finally, in Fig. 14, we display the jet shape obtained with PYTHIA6 for a jet energy $\sim 110 \mathrm{GeV}$ and display the role of hadronization between a fictitious partonic shower and a hadronic shower. For the hadronic shower the study includes all particles in an event. The shift due to the role of hadronization is very small and can be cross-checked to verify that it is the same as the shift displayed for the energy collimation at $E_{\text {rec }} \sim 110 \mathrm{GeV}$ in Fig. 11. However, for energy scales lower than $400 \mathrm{GeV}$, the partonic jet shape obtained from PYTHIA6 is slightly closer to the theoretical calculations. From the comparison displayed in Fig. 11 we can conclude that for high-energy jets the shift between both curves is very small and vanishes asymptotically. This is another part of the reason why, PYTHIA6 and YAJEM+BW with account of hadronization provide a more accurate agreement with the data.

\section{SUMMARY}

In this paper, we studied the energy collimation of gluon and quark jets produced in heavy-ion collisions and the jet shape of hadrons produced in $p p$ and $\mathrm{PbPb}$ collisions at 2.76 TeV. We extracted the LLA and NLLA jet shapes for quark and gluon jets from the jet energy collimation in the frame of DGLAP evolution at large $x$ including the scaling violation of FFs for the first time in this work. More efforts in the numerical framework are however required in order to improve our results and provide a more accurate description which may improve the shape and normalization of both observables, as explained in the main body of this paper.

The NLLA energy collimation seems to capture a more complete analytical description of this observable than the LLA energy collimation obtained in Ref. [33], particularly in gluon jets, but a disagreement with YAJEM+BW and the data still persists, which is more pronounced in more biased quark showers with smaller jet resolutions, i.e. $R=0.1$. The difference between gluon and quark jets for this observable is qualitatively well described by both 
medium-modified NLLA and YAJEM+BW descriptions, i.e. both provide stronger energy collimation in quark jets than in gluon jets and the NLLA description improves the normalization for partons carrying the intermediate energy fractions $x \sim 0.5$. Though this quantity cannot be directly measured for each type of jet separately, their combination would lead to the quantification of the jet broadening at high-energy heavy-ion experiments, i.e. the NLLA formula in the vacuum $\left(N_{s}=1\right)$ and PYTHIA6 predict a much faster increase of the energy collimation than the mediummodified NLLA $\left(N_{s}=1.4\right)$ and YAJEM+BW calculations as the energy scale increases.

We extracted the jet shape from the analysis performed for the energy collimation and compared the NLLA, LLA, PYTHIA6 and YAJEM+BW calculations with the CMS $p p$ and $\mathrm{PbPb}$ data at $2.76 \mathrm{TeV}$. The final biased and unbiased comparison for this observable clearly shows the importance of taking all jet-finding conditions into account in order to get as accurate results as possible in the comparison of Monte Carlo event generators and theoretical predictions with the data.

The NLLA and LLA predictions qualitatively describe the jet shapes but fail to reproduce the right normalization of this observable. The reasons for this disagreement are the same as those previously presented for the energy collimation in the last paragraph of Sec. III D. The biased jet shape provided by PYTHIA6 is in very good agreement with $p p$ CMS data and the medium-modified biased jet shape from YAJEM+BW qualitatively describes the sub-jet broadening shown by $\mathrm{PbPb} \mathrm{CMS}$ data for larger values of $r$, although it is much weaker in CMS data than in the YAJEM+BW result. Gluon jets produce a wider shower broadening than quark jets but they get even more suppressed by biases than quark jets, which clearly dominate the data for $E_{\text {rec,jet }} \geq 100 \mathrm{GeV}$. This new example proves that biases appear to strongly suppress the relevant physics of jet quenching we want to understand and hence, information is lost concerning the early stage of jet evolution and its interaction with the medium in the study of this observable; indeed, the trigger bias suppresses the range of possible medium modifications brought by the medium-induced soft gluon radiation [55].

Of course our results for the jet shape and comparison with the data reflect the characteristics of the BW prescription and hence should be compared and improved with calculations using other models or more conveniently the ongoing calculations of Refs. [25,29] (for an interesting review see also Ref. [56]); a comparison with YAJEM-DE [57] may for instance be desirable.

\section{ACKNOWLEDGMENTS}

R. P.-R. is grateful to Beomsu Chang, DongJo Kim and Norbert Novitzky for their expert support on the Monte Carlo analysis and coding techniques, and to Wolfgang Ochs for useful discussions and comments on the manuscript. We acknowledge support from the Academy researcher program of the Academy of Finland, Project No. 130472.
[1] D. d'Enterria, arXiv:0902.2011.

[2] M. Gyulassy and X.-N. Wang, Nucl. Phys. B420, 583 (1994).

[3] R. Baier, Y. L. Dokshitzer, A. H. Mueller, S. Peigne, and D. Schif, Nucl. Phys. B484, 265 (1997).

[4] B. G. Zakharov, JETP Lett. 65, 615 (1997).

[5] K. Adcox et al., Phys. Rev. Lett. 88, 022301 (2001).

[6] S. S. Adler et al., Phys. Rev. Lett. 91, 072303 (2003).

[7] A. Adare et al., Phys. Rev. Lett. 101, 162301 (2008).

[8] S. Chatrchyan et al. (CMS Collaboration), Phys. Lett. B 730, 243 (2014).

[9] A. Adare et al., Phys. Rev. Lett. 111, 032301 (2013).

[10] Y. L. Dokshitzer, Sov. Phys. JETP 46, 641 (1977).

[11] V. N. Gribov and L. N. Lipatov, Sov. J. Nucl. Phys. 15, 438 (1972).

[12] G. Altarelli and G. Parisi, Nucl. Phys. B126, 298 (1977).

[13] M. Bengtsson and T. Sjostrand, Phys. Lett. B 185, 435 (1987).

[14] E. Norrbin and T. Sjostrand, Nucl. Phys. B603, 297 (2001).
[15] R. Baier, D. Schiff, and B. G. Zakharov, Annu. Rev. Nucl. Part. Sci. 50, 37 (2000).

[16] C. A. Salgado and U. A. Wiedemann, Phys. Rev. Lett. 89, 092303 (2002).

[17] M. Gyulassy, P. Lévai, and I. Vitev, Phys. Lett. B 538, 282 (2002).

[18] E. Wang and X.-N. Wang, Phys. Rev. Lett. 89, 162301 (2002).

[19] M. Shimomura, Nucl. Phys. A774, 457 (2006).

[20] S. Afanasiev et al., Phys. Rev. C 80, 054907 (2009).

[21] D. Magestro, Nucl. Phys. A774, 573 (2006).

[22] J. Adams et al., Phys. Rev. Lett. 97, 162301 (2006).

[23] Y. Mehtar-Tani, C. A. Salgado, and K. Tywoniuk, Phys. Lett. B 707, 156 (2012).

[24] Y. Mehtar-Tani, C. A. Salgado, and K. Tywoniuk, Phys. Rev. Lett. 106, 122002 (2011).

[25] J.-P. Blaizot, F. Dominguez, E. Iancu, and Y. Mehtar-Tani, arXiv:1311.5823.

[26] V. A. Khoze and W. Ochs, Int. J. Mod. Phys. A 12, 2949 (1997).

[27] N. Borghini and U. A. Wiedemann, arXiv:hep-ph/0506218.

[28] G. C. Nayak, Phys. Part. Nucl. 43, 742 (2012). 
[29] Y. Mehtar-Tani and K. Tywoniuk, arXiv:1401.8293.

[30] S. Albino, B. A. Kniehl, and R. Perez-Ramos, Nucl. Phys. B819, 306 (2009).

[31] S. Albino, Rev. Mod. Phys. 82, 2489 (2010).

[32] Y. L. Dokshitzer, V. A. Khoze, and S. I. Troian, Int. J. Mod. Phys. A 07, 1875 (1992).

[33] R. Perez-Ramos and V. Mathieu, Phys. Lett. B 718, 1421 (2013).

[34] P. Nason and B. R. Webber, Nucl. Phys. B421, 473 (1994).

[35] T. Renk, Phys. Rev. C 78, 034908 (2008).

[36] M. H. Seymour, Nucl. Phys. B513, 269 (1998).

[37] C. A. Salgado and U. A. Wiedemann, Phys. Rev. Lett. 93, 042301 (2004).

[38] I. Vitev, S. Wicks, and B.-W. Zhang, J. High Energy Phys. 11 (2008) 093.

[39] M. Cacciari, G. P. Salam, and G. Soyez, Eur. Phys. J. C 72, 1896 (2012).

[40] M. Cacciari and G. P. Salam, Phys. Lett. B 641, 57 (2006).

[41] Y. L. Dokshitzer, D. Diakonov, and S. I. Troian, Phys. Rep. 58, 269 (1980).

[42] Y. L. Dokshitzer, V. A. Khoze, A. H. Mueller, and S. I. Troian, Basics of Perturbative QCD (Editions Frontières, Gif-sur-Yvette, France, 1991); Rev. Mod. Phys. 60, 373 (1988).
[43] S. Albino, B. A. Kniehl, G. Kramer, and W. Ochs, Eur. Phys. J. C 36, 49 (2004).

[44] W. Furmanski and R. Petronzio, Phys. Lett. 97B, 437 (1980).

[45] T. Renk, Phys. Rev. C 79, 054906 (2009).

[46] T. Renk, Phys. Rev. C 85, 044903 (2012).

[47] T. Renk, Phys. Rev. C 86, 061901 (2012).

[48] T. Renk, Phys. Rev. C 87, 024905 (2013).

[49] T. Renk, H. Holopainen, R. Paatelainen, and K. J. Eskola, Phys. Rev. C 84, 014906 (2011).

[50] H.-L. Lai, J. Huston, Z. Li, P. Nadolsky, J. Pumplin, D. Stump, and C.-P. Yuan, Phys. Rev. D 82, 054021 (2010).

[51] K. J. Eskola, V. J. Kolhinen, and C. A. Salgado, Eur. Phys. J. C 9, 61 (1999).

[52] J. Gallicchio and M. D. Schwartz, J. High Energy Phys. 04 (2013) 090.

[53] A. H. Mueller, Nucl. Phys. B241, 141 (1984).

[54] K. J. Eskola and H. Honkanen, Nucl. Phys. A713, 167 (2003).

[55] T. Renk, Phys. Rev. C 88, 054902 (2013).

[56] J. Casalderrey-Solana, Y. Mehtar-Tani, C. A. Salgado, and K. Tywoniuk, Phys. Lett. B 725, 357 (2013).

[57] T. Renk, Phys. Rev. C 84, 067902 (2011). 